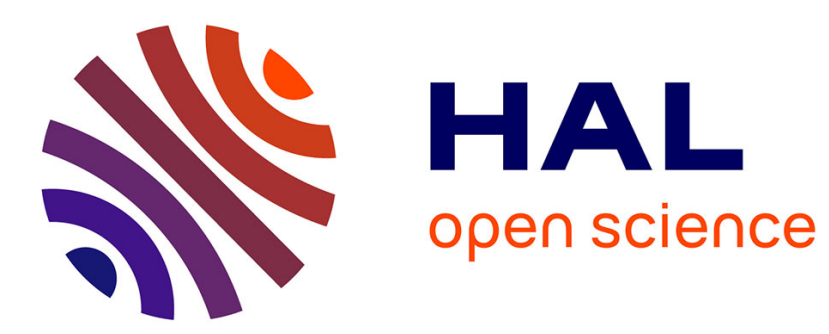

\title{
Randomisation in Automata on Infinite Trees
}

\author{
Arnaud Carayol, Axel Haddad, Olivier Serre
}

\section{To cite this version:}

Arnaud Carayol, Axel Haddad, Olivier Serre. Randomisation in Automata on Infinite Trees. ACM

Transactions on Computational Logic, 2014, 15 (3), pp.24. 10.1145/2629336 . hal-01260669

\section{HAL Id: hal-01260669 \\ https://hal.inria.fr/hal-01260669}

Submitted on 22 Jan 2016

HAL is a multi-disciplinary open access archive for the deposit and dissemination of scientific research documents, whether they are published or not. The documents may come from teaching and research institutions in France or abroad, or from public or private research centers.
L'archive ouverte pluridisciplinaire $\mathbf{H A L}$, est destinée au dépôt et à la diffusion de documents scientifiques de niveau recherche, publiés ou non, émanant des établissements d'enseignement et de recherche français ou étrangers, des laboratoires publics ou privés. 


\title{
Randomisation in Automata on Infinite Trees
}

\author{
ARNAUD CARAYOL \\ LIGM (CNRS \& Université Paris Est) \\ and \\ AXEL HADDAD \\ LIAFA (CNRS \& Université Paris Diderot - Paris 7) \& LIGM (CNRS \& Université Paris \\ Est) \\ and \\ OLIVIER SERRE \\ LIAFA (CNRS \& Université Paris Diderot - Paris 7)
}

\begin{abstract}
We study finite automata running over infinite binary trees. A run of such an automaton over an input tree is a tree labeled by control states of the automaton: the labelling is built in a top-down fashion and should be consistent with the transitions of the automaton. A branch in a run is accepting if the $\omega$-word obtained by reading the states along the branch satisfies some acceptance condition (typically an $\omega$-regular condition such as a Büchi or a parity condition). Finally, a tree is accepted by the automaton if there exists a run over this tree in which every branch is accepting.

In this paper, we consider two relaxations of this definition introducing a qualitative aspect.

First, we relax the notion of accepting run by allowing a negligible set (in the sense of measure theory) of non-accepting branches. In this qualitative setting, a tree is accepted by the automaton if there exists a run over this tree in which almost every branch is accepting. This leads to a new class of tree languages, qualitative tree languages. This class enjoys many good properties: closure under union and intersection (but not under complement), emptiness is decidable in polynomial time. A dual class, positive tree languages, is defined by requiring that an accepting run contains a non-negligeable set of branches.

The second relaxation, is to replace the existential quantification (a tree is accepted if there exists some accepting run over the input tree) by a probabilistic quantification (a tree is accepted if almost every run over the input tree is accepting). For the run, we may use either classical acceptance or qualitative acceptance. In particular, for the latter, we exhibit a tight connection with partial observation Markov decision processes. Moreover, if we additionally restrict to the Büchi condition, we show that it leads to a class of probabilistic automata on infinite trees enjoying a decidable emptiness problem. To our knowledge, this is the first positive result for a class of probabilistic automaton over infinite trees.
\end{abstract}

Categories and Subject Descriptors: F.4.3 [Mathematical Logic and Formal Languages]: Formal Languages - Classes defined by grammars or automata; Decision problems; F.1.1 [Computation by Abstract Devices]: Models of Computation-Automata

General Terms: Theory, Automata, Games

Additional Key Words and Phrases: Finite Automata on Infinite Trees, Measure Theory, (Partial Observation) Markov Decision Processes, Probabilistic Automata

\section{INTRODUCTION}

Roughly speaking a finite automaton on infinite trees is a finite memory machine that takes as input an infinite node-labelled binary tree and processes it in a top- 
down fashion as follows. It starts at the root of the tree in its initial state, and picks (possibly nondeterministically) two successor states, one per son, according to the current control state, the letter at the current node and the transition relation. Then the computation proceeds in parallel from both sons, and so on. Hence a run of the automaton on an input tree is a labelling of this tree by control states of the automaton, that should satisfy the local constrains imposed by the transition relation. A branch in a run is accepting if the $\omega$-word obtained by reading the states along the branch satisfies some acceptance condition (typically an $\omega$-regular condition such as a Büchi or a parity condition). Finally, a tree is accepted by the automaton if there exists a run over this tree in which every branch is accepting. An $\omega$-regular tree language is a tree language accepted by some tree automaton equipped with a parity condition.

Finite automata on infinite trees were originally introduced by Rabin in [Rabin 1969] to prove the decidability of the monadic second order logic (MSOL) over the full binary tree. Indeed, Rabin proved that for any MSOL formula, one can construct a tree automaton that accepts a non empty language if and only if the original formula holds at the root of the full binary tree. These automata were also successfully used by Rabin in [Rabin 1972] to solve Church's synthesis problem [Church 1962], which ask for constructing a circuit based on a formal specification (typically a expressed in monadic second order logic) describing the desired input/output behaviour. His approach was to represent the set of all possible behaviour of a circuit by an infinite tree (directions are used to code the input while node labels along a branch code the output) and to reduce the synthesis problem to emptiness of a tree automaton accepting all those trees coding circuits satisfying the specification.

Since then, automata on infinite trees and their variants have been intensively studied and found many applications, in particular in logic. Connections between automata on infinite trees and logic are discussed in the excellent surveys [Thomas 1997; Vardi and Wilke 2007].

A fundamental result of Rabin is that $\omega$-regular tree languages form a Boolean algebra [Rabin 1969]. The hard part in this proof is the complementation, and since the publication of this result in 1969 , it has been a challenging problem to simplify the proof. A much simpler one was obtained in [Gurevich and Harrington 1982] making use of two-player games on graphs for checking membership of a tree in the language accepted by the automaton: the first player (called Automaton) builds a run on the input tree while the second player (called Pathfinder) tries to exhibit a rejecting branch in the run. Beyond this result, the tight connection between automata and games is one of the main tools in automata theory (see e.g. [Grädel et al. 2002; Löding 2011]).

In this article, we consider variations of the classical model of tree automata over infinite trees. These variations involve, in two different ways, probabilities and preserve the fruitful connection with game theory.

In the first part of this paper, we consider a relaxed notion of an accepting run. While the usual definition requires for a run to be accepting that all branches in it satisfy the acceptance condition, we allow a negligible set of non-accepting branches. In this qualitative setting, a tree is accepted by the automaton if there 
exists a run over this tree in which almost every (in the usual sense from measure theory) branch is accepting. With the parity condition, this leads to a new class of tree languages that we call qualitative tree languages. We show that this class enjoys many desirable properties: closure under union and intersection (but not under complement), emptiness is decidable in polynomial time (contrasting with the fact that no polynomial algorithm is known for the emptiness test of standard parity tree automata). We also prove that there exists a strong connection between automata accepting qualitative tree languages and Markov decision processes, which play here a similar role as two-player games for usual tree automata. We also discuss the positive setting, where a run is accepting if the set of accepting branches in it has a strictly positive measure.

The idea of allowing a certain amount of rejecting branches in a run was already considered in [Beauquier et al. 1991; Beauquier and Niwiński 1995], where it was required that the number of accepting branches in a run belong to a specified set of cardinals $\Gamma$. In particular, they proved that if $\Gamma$ consists of all cardinals greater than some $\gamma$, then one obtains a regular tree language. Qualitative tree languages as defined in this article are not captured by the work of [Beauquier and Niwiński 1995]. Indeed, our classes are incomparable with regular tree languages.

In the second part of this paper, we investigate probabilistic automata on infinite trees. Acceptance by an automaton is based on existential quantification: an input is accepted if there exists an accepting run over it. Probabilistic automata are an alternative way to define acceptance. On finite words they have been introduced by Rabin in [Rabin 1963]. Compared with the standard setting, the non-deterministic guesses are replaced by random choices (according to some probabilistic distribution depending on the control state and the input letter). Hence, the set of transitions is replaced by a probability distribution over the set of all transitions which induces a probability measure on the set of runs of the automaton and acceptation is defined using a threshold $0<\lambda<1$ on the probability of a run to be accepting. In contrast to the non-deterministic setting, the emptiness problem for probabilistic automata on finite words is undecidable [Paz 1971].

The probabilistic model was recently extended to infinite words in [Baier and Größer 2005] by Baier and Größer ${ }^{1}$ and studied in more details in [Baier et al. 2008; Chadha et al. 2009; Chatterjee et al. 2009]. In addition to the threshold criterion, two additional semantics were considered : almost-sure and probable which respectively corresponds to a probability 1 or $>0$ for a run to be accepting ${ }^{2}$. Surprisingly the class of languages defined by Büchi automata with the probable semantics is closed under complement, which implies that it coincides with the class of languages defined by co-Büchi automata with the almost-sure semantics ${ }^{3}$. The

\footnotetext{
${ }^{1}$ A previous attempt by Reisz[Reisz 1999] should be mentioned but this approach does not make real use of probabilities as in this setting an input word is accepted if after some time the behaviour in the run becomes deterministic.

${ }^{2}$ In the finite word case, the almost-sure and probable acceptance are trivial as the set of runs for a given word is finite.

${ }^{3}$ Indeed, let $L$ be accepted by a Büchi automaton with the probable acceptance. As one can complement, there is a Büchi automaton with the probable semantics $\mathcal{A}$ such that $\bar{L}$ is the language accepted by $\mathcal{A}$. If one sees $\mathcal{A}$ as a co-Buchi automaton $\mathcal{B}$ (final states becoming the forbidden ones) with an almost-sure semantics, $\mathcal{B}$ accepts a word if and only if $\mathcal{A}$ does not. Hence, $\mathcal{B}$ recognises
} 
emptiness problem for Büchi automata with the almost-sure semantics as well as for co-Büchi automata with the probable semantics are decidable. However the emptiness problem for Büchi automata with the probable semantics as well as for co-Büchi automata with the almost-sure semantics are undecidable. Of course, emptiness is undecidable when considering a threshold semantics. See [Baier et al. 2012] for a very rich overview of this topic.

In this paper, we consider probabilistic automata on infinite trees. We first focus on the almost-sure semantics, i.e. a tree is accepted if almost every run over it is accepting, and later discuss the probable semantics, i.e. a tree is accepted if the set of accepting runs on it has a (strictly) positive measure. Of course each semantics can be used in combination with the acceptance criteria on runs: the classical one (all branches are accepting), the qualitative one (almost all branches are accepting) and the positive one (there is a non negligible set of accepting branches). For all these combinations, we establish that the definition makes sense (i.e. we prove measurability of the set of accepting runs).

For the qualitative criterion on runs combined with the almost-sure semantics, as well as for the probable criterion on runs combined with the positive semantics, we prove that there exists a strong connection with partial observation Markov decision processes. This condition is independent of the acceptance condition on branches (Büchi, co-Büchi, parity...). In particular, for the Büchi (resp. co-Büchi) acceptance condition on branches, probabilistic automata on infinite trees with the qualitative criterion on runs combined with the almost-sure semantics (resp. with the positive criterion on runs combined with the probable semantics) enjoy a decidable emptiness problem. To our knowledge, this is the first positive result for a class of probabilistic automata over infinite trees. On the negative side, we derive from undecidability results on probabilistic automata on infinite words similar results for probabilistic automata on infinite trees. Note that whilst being immediate for the classical criterion on runs such a reduction is not as simple for the qualitative and positive criteria.

The paper is organised as follows. In Section 2 we introduce general notations and definitions. In Section 3 we define the class of qualitative tree languages, present their properties (closure properties, decidability properties) and their tight connections with Markov decision processes, and establish that they are incomparable with regular tree languages. We also discuss possible variants of the model (leading the notion of positive tree languages). In Section 4 we focus on the probabilistic model. We first justify the validity of the definition and give some examples. Then we provide connections with partial observation Markov decision processes from which we derive positive results for the emptiness problem on a subclass. Finally we discuss variants of the probabilistic model. Section 5 summarises the contributions of the paper.

the complement of $\bar{L}$, namely $L$.

ACM Transactions on Computational Logic, Vol. V, No. N, Month 20YY. 


\section{DEFINITIONS}

\subsection{Words and Trees}

An alphabet $A$ is a finite set of letters. In the sequel $A^{*}$ denotes the set of finite words over $A$, and $A^{\omega}$ the set of infinite words over $A$. The empty word is written $\varepsilon$; the length of a word $u$ is denoted by $|u|$. Let $u$ be a finite word and $v$ be a (possibly infinite) word. Then $u \cdot v$ denotes the concatenation of $u$ and $v$; the word $u$ is a prefix of $v$, denoted $u \sqsubseteq v$, if there exists a word $w$ such that $v=u \cdot w$. We denote by $u \sqsubset v$ the fact that $u$ is a strict prefix of $v$ (i.e. $u \sqsubseteq v$ and $u \neq v$ ). For some word $u$ and some integer $k \geqslant 0$, we denote by $u^{k}$ the word obtained by concatenating $k$ copies of $u$ (with the convention that $u^{0}=\varepsilon$ ).

In this paper we consider full binary node-labelled trees. An A-labelled tree $t$ is a mapping from $\{0,1\}^{*}$ to $A$. In this context, an element $u \in\{0,1\}^{*}$ is called a node, and the node $u \cdot 0$ (resp. $u \cdot 1)$ is the left son (resp. right son) of $u$. The node $\varepsilon$ is called the root. We shall refer to $|u|$ as the depth of $u$. The letter $t(u)$ is called the label of $u$ in $t$.

A branch is an infinite word $\pi \in\{0,1\}^{\omega}$. We write $\mathrm{Br}=\{0,1\}^{\omega}$ for the set of all branches. A node $u$ belongs to a branch $\pi$ if $u$ is a prefix of $\pi$. For an $A$-labelled tree $t$ and a branch $\pi=\pi_{0} \pi_{1} \cdots$ we define the label of $\pi$ as the $\omega$-word $t(\pi)=t(\varepsilon) t\left(\pi_{0}\right) t\left(\pi_{0} \pi_{1}\right) t\left(\pi_{0} \pi_{1} \pi_{2}\right) \cdots$. The cone going through a node $u$ is the set Cone $(u)=u \cdot\{0,1\}^{\omega}$. A sub-cone of a cone Cone $(u)$ is a cone Cone $(v)$ with $u \sqsubseteq v$.

Given a tree $t$ and a node $u$, the subtree of $\boldsymbol{t}$ rooted at $\boldsymbol{u}$ denoted $t[u]$ is the tree defined by $t[u](v)=t(u \cdot v)$. A tree $t$ is said to be regular if it contains only finitely many different subtrees, i.e. the set $\left\{t[u] \mid u \in\{0,1\}^{*}\right\}$ is finite.

We assume that the reader is familiar with basic notions of measure theory and from probability theory and we use [Bauer 2001; 1996] as references for all known results related to this field. Let $\mathcal{F}_{\mathrm{Br}}$ be the $\sigma$-algebra generated by the set of cones (i.e. the smallest set of subsets of $\{0,1\}^{\omega}$ containing the cones and closed under countable union and complementation). Let $\mu$ be the unique probability measure on $\mathcal{F}_{\mathrm{Br}}$ such that for all $u \in\{0,1\}^{*}, \mu(\operatorname{Cone}(u))=2^{-|u|}$. The existence and uniqueness of $\mu$ are guaranteed by Carathéodory's extension theorem [Bauer 2001, Theorem 5.6, p. 24]. For all $0<p<1$, a probability measure $\mu_{p}$ is similarly defined by taking $\mu_{p}(\operatorname{Cone}(u))=p^{|u|_{0}}(1-p)^{|u|_{1}}$ where $|u|_{0}$ and $|u|_{1}$ respectively designate the number of occurrences of 0 and 1 in $u$. In particular, the measure $\mu$ corresponds to $\mu_{1 / 2}$.

\subsection{Tree Automata and Regular Tree Languages}

A tree automaton $\mathcal{A}$ is a tuple $\left\langle A, Q, q_{\text {ini }}, \Delta\right.$, Acc $\rangle$ where $A$ is the input alphabet, $Q$ is the finite set of states, $q_{\text {ini }} \in Q$ is the initial state, $\Delta \subseteq Q \times A \times(Q \times Q)$ is the transition relation and Acc $\subseteq Q^{\omega}$ is the acceptance condition. In the following, we use the notation $q \stackrel{a}{\rightarrow}\left(q_{0}, q_{1}\right)$ as a shorthand for $\left(q, a,\left(q_{0}, q_{1}\right)\right) \in \Delta$. An automaton is deterministic if $q \stackrel{a}{\rightarrow}\left(q_{0}, q_{1}\right)$ and $q \stackrel{a}{\rightarrow}\left(q_{0}^{\prime}, q_{1}^{\prime}\right)$ implies $q_{0}=q_{0}^{\prime}$ and $q_{1}^{\prime}=q_{1}^{\prime}$. An automaton is complete if, for all $q \in Q$ and $a \in A$ there is at least one pair $\left(q_{0}, q_{1}\right) \in Q^{2}$ such that $q \stackrel{a}{\rightarrow}\left(q_{0}, q_{1}\right)$.

Given an $A$-labelled tree $t$, a run of $\mathcal{A}$ over $t$ is a $Q$-labelled tree $\rho$ such that the root is labelled by the initial state, i.e. $\rho(\varepsilon)=q_{\text {ini }}$; for all nodes $u,(\rho(u), t(u), \rho(u$. $0), \rho(u \cdot 1)) \in \Delta$. 
A branch $\pi \in\{0,1\}^{\omega}$ is accepting in the run $\rho$ if $\rho(\pi) \in$ Acc. A run $\rho$ is accepting if all its branches are accepting. Finally, a tree $t$ is accepted if there exists an accepting run of $\mathcal{A}$ over $t$. The set of all trees accepted by $\mathcal{A}$ is denoted $L(\mathcal{A})$.

We consider the following classical acceptance conditions:

-A reachability condition is given by a subset $F \subseteq Q$ of final states by letting $\operatorname{Reach}(F)=Q^{*} F Q^{\omega}$, i.e. a branch is accepting if it contains a final state.

-A Büchi condition is given by a subset $F \subseteq Q$ of final states by letting $\operatorname{Buchi}(F)=$ $\left(Q^{*} F\right)^{\omega}$, i.e. a branch is accepting if it contains infinitely many final states.

-A co-Büchi condition is given by a subset $F \subseteq Q$ of forbidden states by letting $\operatorname{coBuchi}(F)=Q^{*}(Q \backslash F)^{\omega}$, i.e. a branch is accepting it contains finitely many forbidden states.

-A parity condition is given by a colouring mapping Col : $Q \rightarrow \mathbb{N}$ by letting Parity $=\left\{q_{0} q_{1} q_{2} \cdots \mid \lim \inf \left(\operatorname{Col}\left(q_{i}\right)\right)_{i}\right.$ is even $\}$, i.e. a branch is accepting if the smallest colour appearing infinitely often is even.

All these conditions are examples of $\omega$-regular acceptance conditions, i.e. Acc is regular set of $\omega$-words [Perrin and Pin 2004].

REMARK 1. The parity condition is expressive enough to capture the general case of an arbitrary $\omega$-regular condition. Indeed it is well known that Acc is accepted by a deterministic parity word automaton. By taking the synchronised product of this automaton with the tree automaton, we obtain a parity tree automaton accepting the same language (see e.g. [Perrin and Pin 2004]).

When it is clear from the context, we may replace, in the description of $\mathcal{A}, A c c$ by $F$ (for a reachability, Büchi or co-Büchi condition) or Col (for a parity condition), and we shall refer to the automaton as a reachability (resp. Büchi, co-Büchi, parity) tree automaton. A set $L$ of trees is a regular language if there exists a parity tree automaton $\mathcal{A}$ such that $L=L(\mathcal{A})$. The class of regular tree languages is robust, as illustrated by the following statement.

THEOREM 2. [Rabin 1969],[Emerson et al. 1993] The class of regular tree languages is a Boolean algebra.

A regular tree language is non-empty if and only if it contains a regular tree. Testing the emptiness of a regular tree language (defined by a given parity automaton) is in $\mathrm{NP} \cap$ coNP.

\subsection{Markov Decision Process}

2.3.1 Perfect Information Setting. A probability distribution over a countable set $X$ is a mapping $d: X \rightarrow[0,1]$ such that $\sum_{x \in X} d(x)=1$. In the sequel we denote by $\mathcal{D}(X)$ the set of probability distributions over $X$. In this paper, all probabilities will be rational numbers, which will be described in binary when dealing with encoding.

An arena is a tuple $\mathcal{G}=\left\langle S, s_{\text {ini }}, \Sigma, \zeta\right\rangle$ where $S$ is a countable set of states, $s_{\text {ini }}$ is an initial state, $\Sigma$ is a finite set of actions and $\zeta: S \times \Sigma \rightarrow \mathcal{D}(S)$ is the transition (total) function.

ACM Transactions on Computational Logic, Vol. V, No. N, Month 20YY. 
A play in such an arena proceeds as follows. It starts in state $s_{\text {ini }}$ and Élö̈se picks an action $\sigma$, and a successor state is chosen according to the probability distribution $\zeta\left(s_{\text {ini }}, \sigma\right)$. Then Éloïse chooses a new action and the state is updated and so on forever. Hence a play is an infinite sequence $s_{0} s_{1} s_{2} \cdots \in S^{\omega}$ such that $s_{0}=s_{\text {ini }}$ and for every $i \geqslant 0$, there exists a $\sigma \in \Sigma$ with $\zeta\left(s_{i}, \sigma\right)\left(s_{i+1}\right)>0$. In the sequel we refer to a prefix of a play as a partial play and we denote by Plays the set of all plays.

A (pure) strategy ${ }^{4}$ for Élö̈se is a function $\varphi: S^{*} \rightarrow \Sigma$ assigning to every partial play an action. Of special interest are those strategies that do not require memory: a strategy $\varphi$ is memoryless if $\varphi(\lambda \cdot s)=\varphi\left(\lambda^{\prime} \cdot s\right)$ for all partial play $\lambda, \lambda^{\prime}$ and all states $s$ (i.e. $\varphi$ only depends on the current state). A play $\lambda=s_{0} s_{1} s_{2} \ldots$ is consistent with a strategy $\varphi$ if $\zeta\left(s_{i}, \varphi\left(v_{0} \cdots v_{i}\right)\right)\left(s_{i+1}\right)>0$, for all $i \geqslant 0$.

Now, for any partial play $\lambda$, the cylinder for $\lambda$ is the set $\operatorname{Cyl}(\lambda)=\lambda S^{\omega} \cap$ Plays. Let $\mathcal{F}_{P}$ be the $\sigma$-algebra generated by the set of cylinders. Then, (Plays, $\left.\mathcal{F}_{P}\right)$ is a measurable space.

A strategy $\varphi$ induces a probability space over (Plays, $\mathcal{F}_{P}$ ) as follows: one defines a measure $\mu_{\varphi}$ on cylinders and then uniquely extends it to a probability measure on $\mathcal{F}_{P}$ using the Carathéodory's unique extension theorem. For this, we first define inductively $\mu_{\varphi}$ on cylinders:

—as all plays start from $s_{\text {ini }}$, we let $\mu_{\varphi}\left(\mathrm{Cyl}\left(s_{\text {ini }}\right)\right)=1$;

— for any partial play $\lambda$ ending in some state $s$, we let $\mu_{\varphi}\left(\operatorname{Cyl}\left(\lambda \cdot s^{\prime}\right)\right)=\mu_{\varphi}(\operatorname{Cyl}(\lambda))$. $\zeta(s, \varphi(\lambda))\left(s^{\prime}\right)$.

We also denote by $\mu_{\varphi}$ the unique extension of $\mu_{\varphi}$ to a probability measure on $\mathcal{F}$. Then (Plays, $\mathcal{F}_{P}, \mu_{\varphi}$ ) is a probability space.

An objective is a measurable set $\mathcal{O} \subseteq$ Plays: a play is winning if it belongs to $\mathcal{O}$. A Markov decision process (MDP, aka one-and-half-player game) is a pair $\mathbb{G}=(\mathcal{G}, \mathcal{O})$ where $\mathcal{G}$ is an arena and $\mathcal{O}$ is an objective. In the sequel we should focus on $\omega$-regular objectives (which are easily seen to be measurable), whose definitions are the same as for the acceptance condition on tree automata (the only differences is that we may have an infinite set of states and that we restrict ourselves to the set Plays).

A strategy $\varphi$ is almost-surely winning (resp. positively winning) if $\mu_{\varphi}(\mathcal{O})=$ 1 (resp. $\mu_{\varphi}(\mathcal{O})>0$ ). If such a strategy exists, we say that Éloïse almostsurely wins (resp. positively wins) $\mathbb{G}$. The value of $\mathbb{G}$ is defined as $\operatorname{Val}(\mathbb{G})=$ $\sup _{\varphi} \mu_{\varphi}(\mathcal{O})$, and a strategy $\varphi$ is optimal if $\operatorname{Val}(\mathbb{G})=\mu_{\varphi}(\mathcal{O})$.

When the set of actions $\Sigma$ is reduced to one element, the $\operatorname{MDP}(\mathcal{G}, \mathcal{O})$ is called a Markov chain and we omit the unique action from all the definitions. The set Plays is called the set of traces of the Markov chain and is denoted Traces. We write $\mu_{\mathcal{G}}$ the probability measure associated with the unique strategy. We say that the Markov chain almost-surely fulfils its objective if $\mu_{\mathcal{G}}(\mathcal{O})=1$.

MDPs over finite arenas enjoy many good properties.

Theorem 3. [Courcoubetis and Yannakakis 1990; Chatterjee et al. 2004] Let $\mathbb{G}$ be an MDP over a finite arena with a parity objective. Then, one can decide

${ }^{4}$ We do not consider here randomised strategies as in the setting of this paper they are useless. Note that for finite MDP, optimal strategies - when exists - can always be chosen to be pure. 
in polynomial time whether Élö̈se almost-surely (resp. positively) wins. Moreover, Éloïse always has an optimal memoryless strategy.

2.3.2 Imperfect Information Setting. Now we consider the case where Éloïse has imperfect information about the current state. For this, we consider an equivalence relation $\sim$ over $S$. We let $[s] \sim$ be the equivalence class of $s$ for $\sim$ and $S / \sim$ be the set of equivalence classes of $\sim$ over $S$.

The intuitive meaning of $\sim$ is that two states $s_{1} \sim s_{2}$ cannot be distinguished by Éloïse. We easily extend $\sim$ to partial plays: $s_{0} s_{1} \cdots s_{n} \sim s_{0}^{\prime} s_{1}^{\prime} \cdots s_{n}^{\prime}$ if and only if $s_{i} \sim s_{i}^{\prime}$ for all $i=0, \cdots, n$. As two equivalent plays $\lambda_{1} \sim \lambda_{2}$ cannot be distinguished by Éloïse she should therefore behave the same in both of them.

Hence, we should only consider so-called observation-based strategies. An $\boldsymbol{o b}$ servation-based (pure) strategy is a function $\varphi:(S / \sim)^{*} \rightarrow \Sigma$, i.e., to choose her next action, Éloïse considers the sequence of observations she got so far ${ }^{5}$. In particular, an observation-based strategy $\varphi$ is such that $\varphi(\lambda)=\varphi\left(\lambda^{\prime}\right)$ whenever $\lambda \sim \lambda^{\prime}$. In this context, a memoryless strategy is a function from $S / \sim \rightarrow \mathcal{D}(\Sigma)$, i.e. it only depend on the current equivalence class.

A partial observation Markov decision process (POMDP, aka one-andhalf-player imperfect information game) is a triple $(\mathcal{G}, \sim, \mathcal{O})$ where $\mathcal{G}$ is an arena, $\sim$ is an equivalence relation over states and $\mathcal{O}$ is an objective. We say that Élö̈se almost-surely wins (resp. positively wins) $\mathbb{G}$ if she has an almost-surely (resp. positively) winning observation-based strategy. Finally the value of $\mathbb{G}$ is defined as $\operatorname{Val}(\mathbb{G})=\sup _{\varphi} \mu_{\varphi}(\mathcal{O})$ where $\varphi$ ranges over observation-based strategies; optimality is defined as previously.

The following decidability results are known for POMDP:

Theorem 4. [Baier et al. 2008] In a POMDP with a Büchi (resp. co-Büchi) objective, deciding whether Éloïse almost-surely (resp. positively) wins is ExpTimecomplete. Moreover if Éloïse has an almost-surely (resp. positively) winning strategy, she has an almost-surely (resp. positively) winning strategy with finite memory.

In a POMDP with a co-Büchi (resp. Büchi) objective, it is undecidable whether Éloïse almost-surely (resp. positively) wins.

REMARK 5. The results in Theorem 3 and 4 do not depend on the encoding of probability distributions, as the only relevant information is which probabilities are non zero.

\section{QUALITATIVE TREE LANGUAGES}

\subsection{Definitions}

In the classical definition, a run of a tree automaton $\mathcal{A}$ is accepting if all its branches satisfy the acceptance condition. In this article, we introduce a more relaxed notion of acceptation: a run is qualitatively accepting if almost every (in the sense of the measure $\mu$ ) branch in it is accepting. More formally, consider a tree automaton $\mathcal{A}$ with an $\omega$-regular acceptance condition Acc. A run $\rho$ of $\mathcal{A}$ is qualitatively accepting if the set $\operatorname{AccBr}(\rho)=\left\{\pi \in\{0,1\}^{\omega} \mid \rho(\pi) \in \operatorname{Acc}\right\}$ has measure 1, i.e. $\mu(\operatorname{AccBr}(\rho))=1$. Note that, thanks to Proposition 6 below, the set $\operatorname{AccBr}(\rho)$

\footnotetext{
${ }^{5}$ By abuse of notation, we shall write $\varphi\left(s_{0} \cdots s_{n}\right)$ to mean $\varphi\left(\left[s_{0}\right] \sim \cdots\left[s_{n}\right]_{\sim}\right)$
} 
is indeed measurable. A tree $t$ is qualitatively accepted by $\mathcal{A}$ if there exists a qualitatively accepting run of $\mathcal{A}$ over $t$ and the set of all trees qualitatively accepted by $\mathcal{A}$ is denoted $L_{\text {Qual }}(\mathcal{A})$. Finally, a qualitative tree language is a set $L$ of trees such that there exists a parity automaton $\mathcal{A}$ such that $L_{\mathrm{Qual}}(\mathcal{A})=L$.

Proposition 6. Let $\mathcal{A}$ be a tree automaton equipped with an $\omega$-regular acceptance condition, and let $\rho$ be a run of $\mathcal{A}$. The set $\operatorname{AccBr}(\rho)$ is measurable.

Proof. Below is a simple ad-hoc proof of Proposition 6. Nevertheless, one should notice that Corollary 37 (see Section 4.1.2) directly implies Proposition 6.

The reachability case (with final states $F$ ) is obvious as the set $\operatorname{AccBr}(\rho)$ is the (countable) union of all cones $\mathcal{C}(u)$ with $\rho(u) \in F$.

We focus now on the co-Büchi case (with forbidden states $F$ ). Recall that a branch is accepting if and only if there is a node after which there are only states in $Q \backslash F$.

We claim that

$$
\operatorname{AccBr}(\rho)=\bigcup_{u \in\{0,1\}^{*}}\left(\mathcal{C}(u) \backslash \bigcup_{u \sqsubseteq v, \rho(v) \in F} \mathcal{C}(v)\right)
$$

Let $b \in \operatorname{AccBr}(\rho)$ and let $u$ be a node after which all node labels in $b$ belongs to $Q \backslash F$. Then $b \in \mathcal{C}(u)$ and for all $v \sqsupseteq u, \rho(v) \notin F$ : hence, $b$ belongs to $\mathcal{C}(u) \backslash \bigcup_{u \sqsubseteq v, \rho(v) \in F} \mathcal{C}(v)$. Now, let $b \notin \operatorname{AccBr}(\rho): b$ contains infinitely many occurrences of forbidden states. Therefore, for all node $u$ in $b$ there is another node $v$ below $u$ on $b$ that is labelled by a state in $F$, meaning that $b \in \bigcup_{u \sqsubseteq v, \rho(v) \in F} \mathcal{C}(v)$. Equivalently, $b \notin \mathcal{C}(u) \backslash \bigcup_{u \sqsubseteq v, \rho(v) \in F} \mathcal{C}(v)$. Therefore the set $\operatorname{AccBr}(\rho)$ is measurable. The parity conditions follows from the previous case as one can express them as a Boolean combination of co-Büchi conditions. The general case of an arbitrary $\omega-$ regular condition is obtained as follows. First, one considers a deterministic parity word automaton recognising Acc, and then takes the synchronised product of this automaton with the tree automaton. This leads to a parity tree automaton accepting the same language and whose runs are in bijection with the ones of the original automaton. Moreover as this bijection preserves the set of accepting branches one can conclude from the parity case.

EXAMPLE 7. Let $\mathcal{L}_{a}$ be the language of $\{a, b\}$-labelled trees whose set of branches containing at least one a has measure 1. This language is recognised by the following reachability deterministic automaton $\mathcal{A}=\left\langle\{a, b\},\left\{q_{\mathrm{ini}}, q_{f}\right\}, q_{\mathrm{ini}}, \Delta,\left\{q_{f}\right\}\right\rangle$ where: $\Delta=\left\{q_{\text {ini }} \stackrel{b}{\rightarrow}\left(q_{\text {ini }}, q_{\text {ini }}\right), q_{\text {ini }} \stackrel{a}{\rightarrow}\left(q_{f}, q_{f}\right), q_{f} \stackrel{a}{\rightarrow}\left(q_{f}, q_{f}\right), q_{f} \stackrel{b}{\rightarrow}\left(q_{\text {ini }}, q_{\text {ini }}\right)\right\}$.

If one considers $\mathcal{A}$ as a Büchi automaton, the accepted language consists of those trees whose set of branches containing infinitely many a has measure 1.

EXAMPLE 8. Let $\mathcal{L}_{1}$ be the language of trees $t$ such that in almost every branch, there is a node $u$ labelled by a such that the subtree $t[u]$ has only a on its leftmost branch. This language is recognised by the non-deterministic reachability automaton $\mathcal{A}=\left\langle A, Q, q_{w}, \Delta,\left\{q_{a c c}\right\}\right\rangle$ with $A=\{a, b\}, Q=\left\{q_{w}, q_{l}, q_{a c c}, q_{r e j}\right\}$, and $\Delta$ contains the following transitions: $q_{w} \stackrel{*}{\rightarrow}\left(q_{w}, q_{w}\right), q_{w} \stackrel{a}{\rightarrow}\left(q_{l}, q_{a c c}\right), q_{l} \stackrel{a}{\rightarrow}\left(q_{l}, q_{a c c}\right), q_{l} \stackrel{b}{\rightarrow}$ $\left(q_{r e j}, q_{r e j}\right), q_{a c c} \stackrel{*}{\rightarrow}\left(q_{a c c}, q_{a c c}\right), q_{r e j} \stackrel{*}{\rightarrow}\left(q_{r e j}, q_{r e j}\right)$ (here $*$ is a shorthand for an arbitrary letter). Intuitively, the automaton can wait in state $q_{w}$ as long as it wants. 
Using the second transition, the automaton can guess that the node u (labeled by a) has a leftmost branch containing only a. This assumption is checked by sending on the leftmost branch the state $q_{l}$ and the accepting state $q_{a c c}$ on all other branches. As long as the nodes are labelled by a state $q_{l}$ is propagated to the left son. If all nodes on the leftmost branch starting at $u$ are labelled by a, this branch will be rejecting, but this does not affect the measure as there are only countably many such branches). If a node $v$ labelled by $b$ is encountered in state $q_{l}$ the non-accepting state $q_{r e j}$ is propagated on all branches. This last scenario cannot occur in an accepting run as these cones of rejecting branches have a strictly positive measure. Hence the automaton is penalised for wrong guesses.

For the same reasons as for regular tree languages (cf. Remark 1), the parity condition is expressive enough to capture any $\omega$-regular conditions: for any automaton $\mathcal{A}$ with an $\omega$-regular acceptance condition, there exists a parity automaton $\mathcal{B}$ such that $L_{\text {Qual }}(\mathcal{A})=L_{\text {Qual }}(\mathcal{B})$.

Thanks to the following proposition, we can only focus on complete automata.

Proposition 9. For any tree automaton $\mathcal{A}$ with an $\omega$-regular acceptance condition, there exists a complete tree automaton $\mathcal{B}$ with the same acceptance condition and such that $L_{\mathrm{Qual}}(\mathcal{A})=L_{\mathrm{Qual}}(\mathcal{B})$.

Proof. Let $\mathcal{A}=\left\langle A, Q, q_{\text {ini }}, \Delta\right.$, Acc $\rangle$ be a possibly incomplete automaton. Define a complete automaton $\mathcal{B}=\left\langle A, Q^{\prime}=Q \uplus\left\{q_{\mathrm{rej}}\right\}, q_{\text {ini }}, \Delta^{\prime}\right.$, Acc $\rangle$, where the set $\Delta^{\prime}$ is $\Delta$ augmented with $\left\{q \stackrel{a}{\rightarrow}\left(q_{\mathrm{rej}}, q_{\mathrm{rej}}\right) \mid \nexists \underset{q}{\rightarrow} \stackrel{a}{\rightarrow}\left(q_{0}, q_{1}\right) \in \Delta\right\} \cup\left\{q_{\mathrm{rej}} \stackrel{a}{\rightarrow}\left(q_{\mathrm{rej}}, q_{\mathrm{rej}}\right) \mid a \in A\right\}$; i.e. we add a transition to the sink state $q_{\text {rej }}$ whenever a transition is missing.

Note that as the acceptance condition is unchanged, a branch going through $q_{\text {rej }}$ is rejecting. In particular, as $q_{\text {rej }}$ is a sink state, a run that contains $q_{\text {rej }}$ as a rejecting cone, hence is rejecting (as the measure of the rejecting cone is strictly positive). Therefore, the only accepting runs of $\mathcal{B}$ are actually runs that only uses transitions in $\mathcal{A}$, hence are runs of $\mathcal{A}$. Therefore $L_{\text {Qual }}(\mathcal{A})=L_{\text {Qual }}(\mathcal{B})$.

Unsurprisingly determinism is a restriction.

Proposition 10. There is a qualitative tree language that cannot be qualitatively accepted by any deterministic automaton.

Proof. Let $\mathcal{L}_{\mathrm{a}}$ be the qualitative language (see Example 7) of $\{\mathrm{a}, \mathrm{b}\}$-labelled trees whose set of branches containing at least one a has measure 1. Consider now the language $\mathcal{L}_{\mathrm{a}}^{\prime}=\left\{t \mid t[0] \in \mathcal{L}_{\mathrm{a}}\right.$ or $\left.t[1] \in \mathcal{L}_{\mathrm{a}}\right\}$. Clearly $\mathcal{L}_{\mathrm{a}}^{\prime}$ is qualitative (it suffices to guess one subtree and check that it belongs to $\mathcal{L}_{\mathrm{a}}$ while accepting without any further consideration the other subtree). By contradiction assume that there is a deterministic automaton $\mathcal{A}$ that qualitatively accepts $\mathcal{L}_{\mathrm{a}}^{\prime}$. Indeed, consider the two trees $t_{\mathrm{a}, \mathrm{b}}$ and $t_{\mathrm{b}, \mathrm{a}}$ defined as follows: $t_{\mathrm{a}, \mathrm{b}}(\varepsilon)=t_{\mathrm{b}, \mathrm{a}}(\varepsilon)=\mathrm{b}$, the left subtree of the root of $t_{\mathrm{a}, \mathrm{b}}\left(\right.$ resp. $\left.t_{\mathrm{b}, \mathrm{a}}\right)$ has only a's (resp. b's) and the right subtree of the root of $t_{\mathrm{a}, \mathrm{b}}\left(\right.$ resp. $\left.t_{\mathrm{b}, \mathrm{a}}\right)$ has only b's (resp. a's). Both $t_{\mathrm{a}, \mathrm{b}}$ and $t_{\mathrm{b}, \mathrm{a}}$ belongs to $\mathcal{L}_{\mathrm{a}}^{\prime}$. The run of $\mathcal{A}^{\prime}$ on $t_{\mathrm{a}, \mathrm{b}}$ start with the same transition at the root, and therefore one can combine them and get a run for the tree $t_{\mathrm{b}}$ whose nodes are all labeled by $\mathrm{b}$, which leads a contradiction as $t_{\mathrm{b}} \notin \mathcal{L}_{\mathrm{a}}^{\prime}$. 


\subsection{On the Choice of Measure $\mu$}

The choice of the measure $\mu$ though natural is arbitrary. Considering the measure $\mu_{p}$ for some $0<p<\frac{1}{2}$ would not affect the results obtained in this article (provided that definitions of the games are modified accordingly). However note that changing the measure does change the accepted language for a given automaton.

Proposition 11. Let $0<p<q<1$ be two reals. Let $\mathcal{A}$ be the (deterministic and complete) automaton of Example 7 . Then, there is a tree $t$ such that $\mu_{p}(\operatorname{Acc}(\mathcal{A}, t))=0$ and $\mu_{q}(\operatorname{Acc}(\mathcal{A}, t))=1$, where $\operatorname{Acc}(\mathcal{A}, t)$ denotes the set of accepting branches for the unique run of $\mathcal{A}$ over $t$.

Proof. First recall that $\mathcal{A}$ accepts those trees whose set of branches containing infinitely many a has measure 1 .

We define the $\{\mathrm{a}, \mathrm{b}\}$-labeled tree $t$ by letting for all $u \in\{0,1\}^{*} t(u)=\mathrm{a}$ if and only if $\mu_{p}(\operatorname{Cone}(u)) \leqslant \mu_{q}(\operatorname{Cone}(u))$.

Let $\varepsilon$ be the maximum among $p, q, 1-p, 1-q$.

Following the proof of Kakutani's theorem [Kakutani 1948] as presented in [Muchnik et al. 1998] and [Bienvenu 2008], we consider the measure $\mu_{r}$ for $r=\frac{p+q}{2}$.

We first establish that for all $w \in\{0,1\}^{*}$,

$$
\mu_{r}(\operatorname{Cone}(w))^{2} \geqslant \mu_{p}(\operatorname{Cone}(w)) \mu_{q}(\operatorname{Cone}(w)) \alpha^{|w|}
$$

where $\alpha=1+\frac{(p-q)^{2}}{4 \varepsilon^{2}}$ (note that $\left.\alpha>1\right)$. Indeed,

$$
\begin{aligned}
\mu_{r}(\operatorname{Cone}(w))^{2}= & \left(\frac{(p+q)^{2}}{4}\right)^{|w|_{0}}\left(\frac{((1-p)+(1-q))^{2}}{4}\right)^{|w|_{1}} \\
= & p^{|w|_{0}} q^{|w|_{0}}\left(1+\frac{(p-q)^{2}}{4 p q}\right)^{|w|_{0}}(1-p)^{|w|_{1}} \\
& (1-q)^{|w|_{1}}\left(1+\frac{((1-p)-(1-q))^{2}}{4(1-p)(1-q)}\right)^{|w|_{1}} \\
= & p^{|w|_{0}} q^{|w|_{0}}\left(1+\frac{(p-q)^{2}}{4 p q}\right)^{|w|_{0}}(1-p)^{|w|_{1}} \\
& (1-q)^{|w|_{1}}\left(1+\frac{(p-q)^{2}}{4(1-p)(1-q)}\right)^{|w|_{1}} \\
\geqslant & p^{|w|_{0}} q^{|w|_{0}} \alpha^{|w|_{0}}(1-p)^{|w|_{1}}(1-q)^{|w|_{1}} \alpha^{|w|_{1}} \\
= & \mu_{p}(\operatorname{Cone}(w)) \mu_{q}(\operatorname{Cone}(w)) \alpha^{|w|}
\end{aligned}
$$

Let $K_{n}$ be the set of words $w \in\{0,1\}^{n}$ such that $\mu_{p}(\operatorname{Cone}(w)) \leqslant \mu_{q}(\operatorname{Cone}(w))$ and let $C_{n}$ be the disjoint union of all Cone $(w)$ for all $w \in K_{n}$.

For all $w \in K_{n}$ we have $\mu_{r}(\operatorname{Cone}(w)) \geqslant \mu_{p}(\operatorname{Cone}(w)) \alpha^{n / 2}$, and for all $w \notin K_{n}$ we have $\mu_{r}(\operatorname{Cone}(w)) \geqslant \mu_{q}(\operatorname{Cone}(w)) \alpha^{n / 2}$.

Hence, $\mu_{p}\left(C_{n}\right)=\sum_{w \in K_{n}} \mu_{p}(\operatorname{Cone}(w))$

$$
\begin{aligned}
& \leqslant \alpha^{-n / 2} \sum_{w \in K_{n}} \mu_{r}(\operatorname{Cone}(w)) \\
& \leqslant \alpha^{-n / 2}
\end{aligned}
$$

ACM Transactions on Computational Logic, Vol. V, No. N, Month 20 YY. 
Similarly, $\mu_{q}\left(C_{n}\right)=1-\mu_{q}\left(\overline{C_{n}}\right)$

$$
\begin{aligned}
& =1-\sum_{w \notin K_{n}}, w \in\{0,1\}^{n} \mu_{q}(\operatorname{Cone}(w)) \\
& \geqslant 1-\alpha^{-n / 2} \sum_{w \notin K_{n}, w \in\{0,1\}^{n}} \mu_{r}(\operatorname{Cone}(w)) \\
& \geqslant 1-\alpha^{-n / 2}
\end{aligned}
$$

By definition of $\mathcal{A}$, we have

$$
\operatorname{Acc}(\mathcal{A}, t)=\bigcap_{k \geqslant 0} \bigcup_{n \geqslant k} C_{n}
$$

For $k \geqslant 1$, we let $D_{k}$ denote the set $\bigcup_{n \geqslant k} C_{n}$.

As for all $k \geqslant 1, \mu_{p}\left(D_{k}\right) \leqslant \sum_{n \geqslant k} \alpha^{-\frac{n}{2}} \leqslant \frac{\alpha^{\frac{-k}{2}}}{1-\alpha^{\frac{-1}{2}}}$, we have $\lim _{k \rightarrow \infty} \mu_{p}\left(D_{k}\right)=0$ (recall that $\alpha>1$ ). Moreover for all $k \geqslant 0, \mu_{q}\left(D_{k}\right)=1$ (as $\alpha>1$ and as for all $\left.n \geqslant k, \mu_{q}\left(D_{k}\right) \geqslant \mu_{q}\left(C_{n}\right) \geqslant 1-\alpha^{-n / 2}\right)$.

As the sequence of sets $\left(D_{k}\right)_{k \geqslant 0}$ is decreasing for the inclusion, we have thanks to [Bauer 2001, Theorem 3.2, p. 10] that:

$$
\left\{\begin{array}{l}
\mu_{p}(\operatorname{Acc}(\mathcal{A}, t))=\lim _{k \rightarrow \infty} \mu_{p}\left(D_{k}\right)=0 \\
\mu_{q}(\operatorname{Acc}(\mathcal{A}, t))=\lim _{k \rightarrow \infty} \mu_{q}\left(D_{k}\right)=1
\end{array}\right.
$$

A more general definition is to associate with any letter a in the alphabet a pair $\left(p_{\mathrm{a}}^{0}, p_{\mathrm{a}}^{1}\right) \in[0,1]^{2}$ with $p_{\mathrm{a}}^{0}+p_{\mathrm{a}}^{1}=1$ and then to define the measure of a cone in a tree $t$ by letting $\mu\left(\operatorname{Cone}\left(u_{1} \cdots u_{n}\right)\right)=p_{t(\varepsilon)}^{u_{1}} p_{t\left(u_{1}\right)}^{u_{2}} \cdots p_{t\left(u_{1} \cdots u_{n-1}\right)}^{u_{n}}$. Intuitively, the node label determines the respective weights of the left and right sons in the definition of the measure. In particular the measure $\mu_{p}$ is the one obtained by letting $\left(p_{\mathrm{x}}^{0}, p_{\mathrm{x}}^{1}\right)=(p, 1-p)$ for all letters $\mathrm{x}$ in the alphabet.

Again, with such a measure the results obtained in this article (provided that definitions of the games are modified accordingly) remains correct.

Remark 12. Following Proposition 11, a natural question is whether the class of qualitative tree languages is the same for each distribution $\mu_{p}$ with $0<p<1$. And the same for the above variant with probability values $p_{a}^{0}, p_{a}^{1}$ for all letters in the alphabet. From the statement of Proposition 11 we conjecture that the answer to these questions is no but we leave it open.

\subsection{Pumping Lemma}

Let $t$ be a tree and $u \in\{0,1\}^{*}$ be a node. A pair $\Delta=(t, u)$ is called a pointed tree. With a pointed tree $\Delta_{1}=\left(t_{1}, u_{1}\right)$ and a tree $t_{2}$, we associate a new tree, $\Delta_{1} \cdot t_{2}$, by plugging $t_{2}$ in $t_{1}$ instead of the subtree rooted at $u_{1}$. Formally, $\Delta_{1} \cdot t_{2}(u)=t_{1}(u)$ if $u_{1}$ is not a prefix of $u$ and $\Delta_{1} \cdot t_{2}(u)=t_{2}\left(u^{\prime}\right)$ if $u=u_{1} u^{\prime}$ for some $u^{\prime} \in\{0,1\}^{*}$. We can also define the product of two pointed trees $\Delta_{1}=\left(t_{1}, u_{1}\right)$ and $\Delta_{2}=\left(t_{2}, u_{2}\right)$ by letting $\Delta_{1} \cdot \Delta_{2}=\left(\Delta_{1} \cdot t_{2}, u_{1} \cdot u_{2}\right)$. Finally, with a pointed tree $\Delta=(t, u)$, we associate a tree $\Delta^{\omega}$ by taking an $\omega$-iteration of the product: $\Delta^{\omega}(v)=t\left(v^{\prime}\right)$ where $v^{\prime}$ is the shortest word s.t. $v=u^{k} v^{\prime}$ for some $k \geqslant 0$.

Qualitative tree languages enjoy a pumping lemma (see Figure 13 for an illustration), which contrasts with regular tree languages. Intuitively, pumping does not change acceptance for the qualitative semantics as it may only introduce a set of 
rejecting branches of measure zero (while for the classical semantics introducing a rejecting branch would make the run rejecting).

Lemma 13. Let $\mathcal{A}$ be an $n$-states parity automaton, $t$ be a tree in $L_{\mathrm{Qual}}(\mathcal{A})$ and $u$ be a node of depth greater that $n$. Then there exists three pointed trees $\Delta_{1}, \Delta_{2}$ and $\Delta_{3}$ such that $t=\Delta_{1} \cdot \Delta_{2} \cdot \Delta_{3} \cdot t[u]$ and $\Delta_{1} \cdot \Delta_{2}^{\omega} \in L_{\text {Qual }}(\mathcal{A})$.
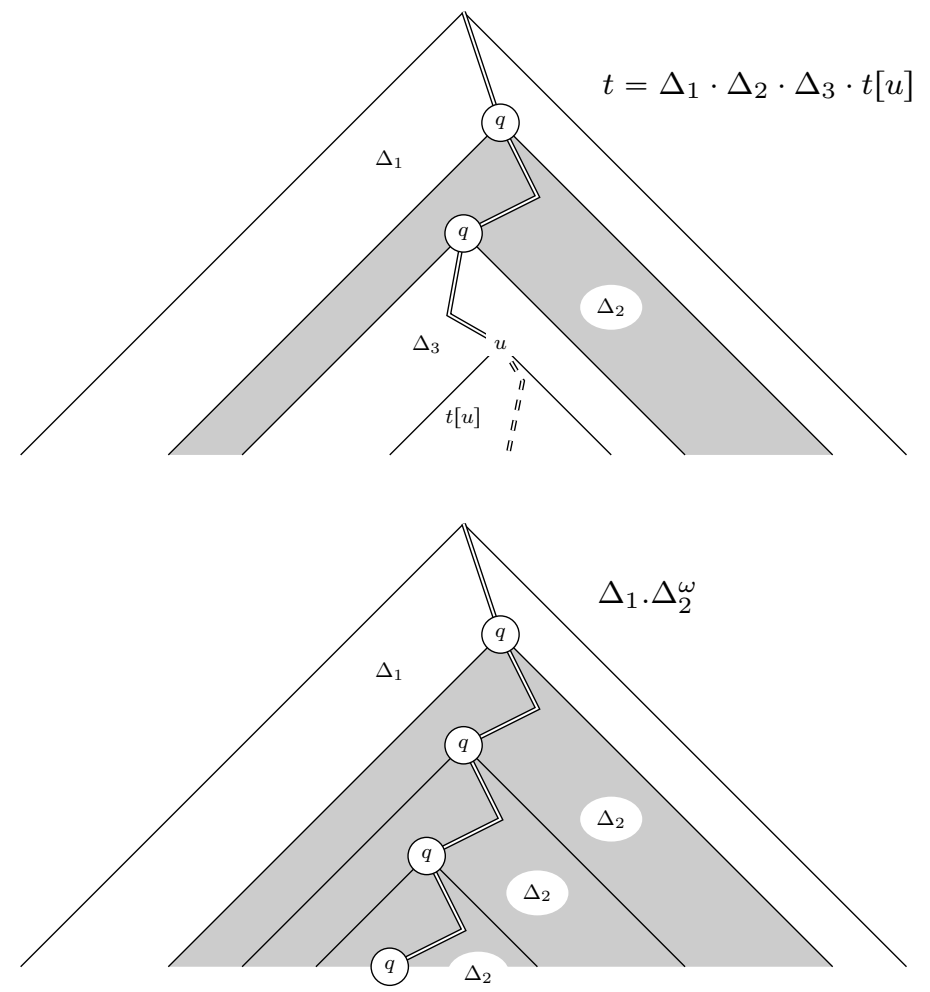

Fig. 1. Pumping Lemma

Proof. Let $\rho$ be an accepting run of $\mathcal{A}$ over $t$. Since $u$ has depth greater than the number of states, there are two nodes $u_{1}$ and $u_{2}$ such that $u_{1} \sqsubseteq u_{2} \sqsubseteq u$ and $\rho\left(u_{1}\right)=\rho\left(u_{2}\right)=q$ for some state $q$. We now decompose $t$ as $t=\Delta_{1} \cdot \Delta_{2} \cdot \Delta_{3} \cdot t[u]$ with $\Delta_{1}=\left(t, u_{1}\right), \Delta_{2}=\left(t\left[u_{1}\right], u_{2}\right)$ and $\Delta_{3}=\left(t\left[u_{2}\right], u\right)$. We decompose $\rho$ in a similar way, by letting $\rho=\Theta_{1} \cdot \Theta_{2} \cdot \Theta_{3} \cdot \rho[u]$ with $\Theta_{1}=\left(\rho, u_{1}\right), \Theta_{2}=\left(\rho\left[u_{1}\right], u_{2}\right)$ and $\Theta_{3}=\left(\rho\left[u_{2}\right], u\right)$. Note that $\Theta_{1} \cdot \Theta_{2}^{\omega}$ is a run of $\mathcal{A}$ over $\Delta_{1} \cdot \Delta_{2}^{\omega}$. A branch in $\Theta_{1} \cdot \Theta_{2}^{\omega}$ can be rejecting for three reasons: it is a rejecting branch in $\rho \backslash \rho\left[u_{1}\right]$; or it is a branch in some of the copies of $\rho\left[u_{1}\right] \backslash \rho\left[u_{2}\right]$; or it is the newly created branch $u_{1} v^{\omega}$ where $v$ is the word such that $u_{2}=u_{1} \cdot v$. As $\rho$ is accepting, the measure of each of these three sets of rejecting branches is 0 (for the second set, it is a finite union of sets of measure 0$)$. Therefore $\Theta_{1} \cdot \Theta_{2}^{\omega}$ is accepting, hence $\Delta_{1} \cdot \Delta_{2}^{\omega} \in L_{\text {Qual }}(\mathcal{A})$. 


\subsection{Closure Properties}

We now investigate the closure properties of qualitative tree languages under Boolean operations.

Proposition 14. Qualitative tree languages are closed under union and intersection.

Proof. The union is for free thanks to non-determinism of the automata model. For the intersection of two languages, $L_{\text {Qual }}\left(\mathcal{A}_{1}\right)$ and $L_{\text {Qual }}\left(\mathcal{A}_{2}\right)$, we consider the usual product automaton whose states set is the Cartesian product of the states set of $\mathcal{A}_{1}$ and of $\mathcal{A}_{2}$, and that simulates $\mathcal{A}_{1}$ (resp. $\mathcal{A}_{2}$ ) on its first (resp. second) component. Hence runs of the product automaton are in bijection with pairs of runs of $\mathcal{A}_{1}$ and $\mathcal{A}_{2}$. The ( $\omega$-regular) acceptance condition requires that the acceptance condition of $\mathcal{A}_{1}$ (resp. $\mathcal{A}_{2}$ ) is fulfilled on the first (resp. second) component. Hence the set of accepting branches in a run of the product automaton is the intersection of the set of accepting branches in the corresponding runs of $\mathcal{A}_{1}$ and $\mathcal{A}_{2}$. Hence, it has measure 1 if and only if both sets have measure 1 , i.e. a run is accepting if and only if both corresponding runs in $\mathcal{A}_{1}$ and $\mathcal{A}_{2}$ are accepting.

Unsurprisingly, qualitative tree languages are not closed under complement. This is a simple consequence of the pumping lemma.

Proposition 15. Qualitative tree languages are not closed under complement.

Proof. Let $\mathcal{L}_{\mathrm{a}}$ be the set of $\{\mathrm{a}, \mathrm{b}\}$-labelled trees whose set of branches containing an a has measure 1. Clearly, $\mathcal{L}_{\mathrm{a}}$ is a qualitative tree language. However, we will show that its complement $\overline{\mathcal{L}_{\mathrm{a}}}$ does not satisfy the pumping lemma, hence is not qualitative. By contradiction assume that $\overline{\mathcal{L}_{\mathrm{a}}}=L_{\text {Qual }}(\mathcal{A})$ for some $n$-state automaton $\mathcal{A}$. Now define a tree $t$ by letting $u=0^{n}$ and $t(v)=\mathrm{b}$ if $v \in t[u]$ and $t(v)=\mathrm{a}$ otherwise. Hence in $t$ a tree is labelled by $\mathrm{a} \mathrm{b}$ if and only if it belongs to the subtree rooted at $u$, and therefore the branches in Cone $(u)$ contains finitely many a, implying that $t \in \overline{\mathcal{L}_{\mathrm{a}}}$. Now, if one uses Lemma 13 for $t$ and $u$, the tree $\Delta_{1} \cdot \Delta_{2}^{\omega}$ (as in the statement of Lemma 13) is the tree whose nodes are all labelled by a, i.e. $\Delta_{1} \cdot \Delta_{2}^{\omega} \notin \overline{\mathcal{L}_{\mathrm{a}}}$, leading a contradiction.

\subsection{Emptiness Problem}

It is well known that tree automata (as acceptors of regular languages) and twoplayer (perfect information) game are closely related [Gurevich and Harrington 1982; Grädel et al. 2002]. In particular, the emptiness problem for regular tree languages and the problem of deciding the winner in a parity game on a finite graph are polynomially equivalent. From the proof of this result also follows that a regular tree language is non-empty if and only if it contains a regular tree.

We show that a similar connection exists between tree automata as acceptors of qualitative tree languages and MDP. For this, fix a parity tree automaton $\mathcal{A}=$ $\left\langle A, Q, q_{\mathrm{ini}}, \Delta, \mathrm{Col}\right\rangle$ and a tree $t$. Consider the arena, depicted in Figure $2, \mathcal{G}_{\mathcal{A}, t}=$ $\left\langle S, s_{\text {ini }}, \Sigma, \zeta\right\rangle$ where $S=Q \times\{0,1\}^{*} \cup\{\perp\}, s_{\text {ini }}=\left(q_{\text {ini }}, \varepsilon\right), \Sigma=\Delta$ and $\zeta$ is defined as follows. First we let $d_{\perp}$ be the distribution defined by $d_{\perp}(s)=1$ if $s=\perp$ and $d_{\perp}(s)=0$ otherwise, and, for all $q_{0}, q_{1} \in Q$ and $u \in\{0,1\}^{*}$, we let $d_{q_{0}, q_{1}, u}$ be the distribution such that $d_{q_{0}, q_{1}, u}\left(q_{0}, u 0\right)=d_{q_{0}, q_{1}, u}\left(q_{1}, u 1\right)=1 / 2$ and $d_{q_{0}, q_{1}, u}(s)=0$ 


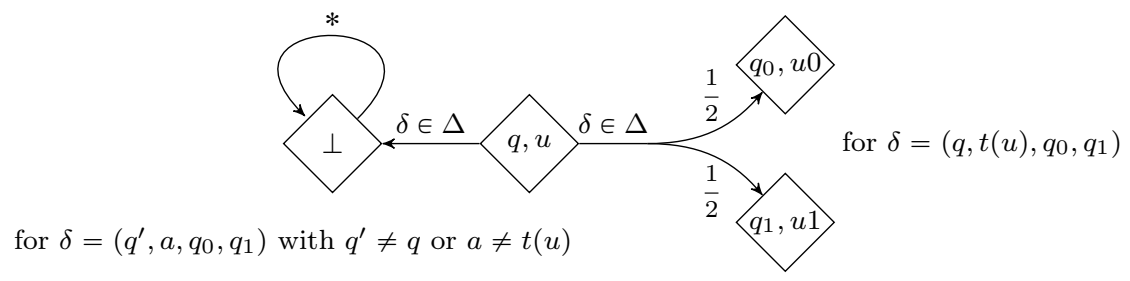

Fig. 2. The arena $\mathcal{G}_{\mathcal{A}, t}$ of the acceptance game.

for all other $s \in S$. Then we let $\zeta\left((q, u),\left(q^{\prime}, a, q_{0}, q_{1}\right)\right)=d_{\perp}$ if $q \neq q^{\prime}$ or $a \neq t(u)$, $\zeta\left((q, u),\left(q, t(u), q_{0}, q_{1}\right)\right)=d_{q_{0}, q_{1}, u}$ and $\zeta(\perp, \sigma)=d_{\perp}$ for all $\sigma \in \Delta$. Finally, we define a colouring function $\rho$ by letting $\rho((q, u))=\operatorname{Col}(q)$ and $\rho(\perp)=1$, and we call $\mathbb{G}_{\mathcal{A}, t}=\left(\mathcal{G}_{\mathcal{A}, t}, \mathcal{O}_{\rho}\right)$ the MDP equipped with the parity objective $\mathcal{O}_{\rho}$ defined by $\rho$.

Then, the following holds:

ThEOREM 16. The tree $t$ belongs to $L_{\mathrm{Qual}}(\mathcal{A})$ if and only if Éloïse almost-surely wins in $\mathbb{G}_{\mathcal{A}, t}$.

Proof. In $\mathbb{G}_{\mathcal{A}, t}$, a partial play that does not visit $\perp$ is a sequence of the form $\left(q_{0}, u_{0}\right)\left(q_{1}, u_{1}\right) \cdots\left(q_{k}, u_{k}\right)$ where for all $i, u_{i+1}=u_{i} x_{i}$ for some $x_{i}=0,1$. Now, with any strategy $\varphi$ of Éloïse we can associate a strategy $\varphi^{\prime}$ of Éloïse that is defined only on those partial plays that are compatible with it (meaning that if in some partial play $\lambda$ Éloïse does not respect $\varphi^{\prime}$ then $\varphi^{\prime}(\lambda)$ is undefined) and coincide with $\varphi$ when defined; it is easily seen that $\varphi$ and $\varphi^{\prime}$ leads the same plays and define the same probability measure. This means that one can only focus on such strategies for Éloïse. We should also only focus on strategies that never reach $\perp$, i.e. it always chooses an action of the form $\left(q, t(u), q_{0}, q_{1}\right)$ if the current state is $(q, u)$. Now, one can remark that for any node $u$ there is a unique partial play $\lambda$ where Éloise respects $\varphi^{\prime}$ and that ends in a state of the form $(q, u)$ for some $q$ such that $\varphi^{\prime}$ is defined on $\lambda$. Therefore, an equivalent way to see $\varphi^{\prime}$ is as a $Q$-labelled tree, that should additionally verify the local properties imposed by $\Delta$, namely the tree should be a run of $\mathcal{A}$ over $t$. It should also be clear that the set of plays where Éloïse respects $\varphi^{\prime}$ is the set of branches in the run associated with $\varphi^{\prime}$ and that this map preserves the measure (in particular the set of winning plays has the same measure as the set of accepting branches in the run), meaning that a strategy with value $\xi$ is mapped to a run whose set of accepting branches has measure $\xi$ and vice-versa. As this map, from strategies to runs is bijective, this concludes the proof: a tree $t$ belongs to $L_{\text {Qual }}(\mathcal{A})$ if and only if there is an accepting run of $\mathcal{A}$ over $t$ if and only if there is an almost surely winning strategy for Éloïse in $\mathbb{G}_{\mathcal{A}, t}$.

Consider the (finite) arena $\mathcal{G}_{\mathcal{A}}=\left\langle S, s_{\text {ini }}, \Sigma, \zeta\right\rangle$, depicted in Figure 3, where $S=Q \times\{0,1\} \cup\left\{q_{\text {ini }}, \perp\right\}, s_{\text {ini }}=q_{\text {ini }}, \Sigma=\Delta$ and $\zeta$ is defined as follows. First we let $d_{\perp}$ be the distribution defined by $d_{\perp}(s)=1$ if $s=\perp$ and $d_{\perp}(s)=0$ otherwise, and, for all $q_{0}, q_{1} \in Q$, we let $d_{q_{0}, q_{1}}$ be the distribution such that $d_{q_{0}, q_{1}}\left(\left(q_{0}, 0\right)\right)=d_{q_{0}, q_{1}}\left(\left(q_{1}, 1\right)\right)=1 / 2$ and $d_{q_{0}, q_{1}}(s)=0$ for all other $s \in S$. Then we let $\zeta\left((q, i),\left(q, a, q_{0}, q_{1}\right)\right)=d_{q_{0}, q_{1}}, \zeta\left((q, i),\left(q^{\prime}, a, q_{0}, q_{1}\right)\right)=d_{\perp}$ if $q \neq q^{\prime}, \zeta\left(q_{\mathrm{ini}},\left(q_{\mathrm{ini}}, a, q_{0}, q_{1}\right)\right)=$ 


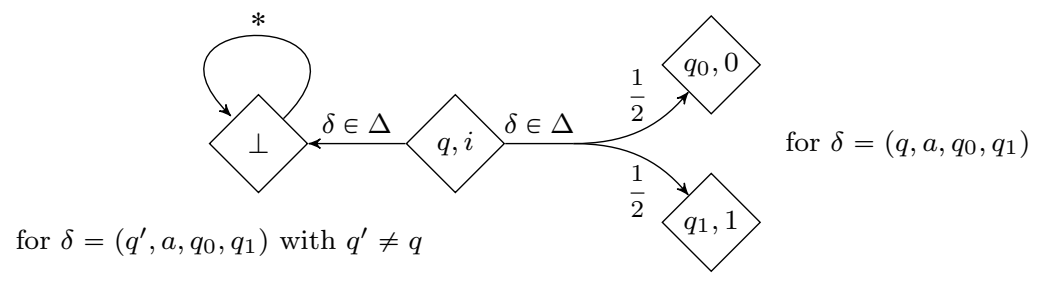

Fig. 3. The arena $\mathcal{G}_{\mathcal{A}}$ of the emptiness game.

$d_{q_{0}, q_{1}}, \zeta\left(q_{\text {ini }},\left(q, a, q_{0}, q_{1}\right)\right)=d_{\perp}$ if $q \neq q_{\text {ini }}$, and $\zeta(\perp, \sigma)=d_{\perp}$ for all $\sigma \in \Delta$. Finally, we define a colouring function $\rho$ by letting $\rho((q, i))=\operatorname{Col}(q)$ and $\rho(\perp)=1$, and we call $\mathbb{G}_{\mathcal{A}}=\left(\mathcal{G}_{\mathcal{A}}, \mathcal{O}_{\rho}\right)$ the MDP equipped with the parity objective $\mathcal{O}_{\rho}$ defined by $\rho$. Then, the following hold:

TheOREM 17. The language $L_{\mathrm{Qual}}(\mathcal{A})$ is non empty if and only if Éloïse almostsurely wins in $\mathbb{G}_{\mathcal{A}}$ from $q_{\mathrm{ini}}$.

Proof. Following the same lines as for the proof of Theorem 16 it is easily seen that strategies that avoid state $\perp$ are in bijection with pairs of $(t, \rho)$ where $t$ is an $A$-labeled tree and $\rho$ is a run of $\mathcal{A}$ over $t$. Moreover this map preserves the measure, meaning that a strategy with value $\xi$ is mapped to a pair $(t, \rho)$ such that the set of accepting branches in $\rho$ has measure $\xi$. In particular, Éloïse almost surely wins in $\mathbb{G}_{\mathcal{A}}$ if and only if there is a tree $t$ that is qualitatively accepted by $\mathcal{A}$.

COROllary 18. Let $\mathcal{A}$ be a parity tree automaton. Then one can decide whether $L_{\mathrm{Qual}}(\mathcal{A})=\varnothing$ in polynomial time. Moreover, if $L_{\mathrm{Qual}}(\mathcal{A}) \neq \varnothing$, it contains a regular tree, and such a tree can be constructed in polynomial time.

Proof. Emptiness in polynomial time follows from theorems 17 and 3. Now, if Éloïse has an almost surely strategy, then she has a memoryless one (Theorem 3 ). Then the tree and the run associated with this strategy are regular, the run is qualitatively accepting. As one can compute (when exists) an almost surely winning positional strategy, one can also compute (when exists) in polynomial time a regular tree in $L_{\text {Qual }}(\mathcal{A})$.

REMARK 19. Motivated by a decision problem for qualitative tree language we designed a polynomial reduction of the emptiness problem to the problem of deciding almost-surely winning in a finite MDP.

As already mentioned, a similar connection exists between tree automata (as acceptors of regular tree languages) and two-player (perfect information) games (see e.g. [Gurevich and Harrington 1982; Grädel et al. 2002]). Indeed, the emptiness problem for regular tree languages and the problem of deciding the winner in a parity game on a finite graph are polynomially equivalent.

Hence, one may ask whether conversely the problem of deciding almost-surely winning in a finite MDP can be polynomially reduced to the emptiness problem for qualitative tree languages. It is indeed possible and the proof is very similar to the one from a two-player game to a regular tree language. We briefly sketch the proof below.

ACM Transactions on Computational Logic, Vol. V, No. N, Month 20YY. 
First note that one can, up to coding, restrict its attention to finite MDP with only two actions and such that from any state and any action there are always two possible successors that can both be reached with same probability $1 / 2$. Then one designs a deterministic tree automaton whose states are identified with the ones of the MDP, whose input alphabet is identified with the set of actions of the MDP, and whose transition function mimics the one of the MDP. Then one concludes by noting that there is a bijection between trees and strategies and that this bijection is such that the measure of the accepting branches in the (unique) run of the automaton on a tree equals the value of the corresponding strategy in the MDP.

\subsection{Regular Tree Languages and Qualitative Tree Languages are Incomparable}

In this section, we prove that regular tree languages and qualitative tree languages are incomparable.

Proposition 20. There is a regular tree language that is not qualitative.

Proof. Consider the regular tree language $L$ of those $\{a, b\}$-labelled trees that contains at least one node labeled by b. By contradiction, assume that $L=$ $L_{\text {Qual }}(\mathcal{A})$ for some $n$-state automaton $\mathcal{A}$ and let $t \in L$ be the tree defined by $t(u)=\mathrm{b}$ if $u=0^{n}$ and $t(u)=\mathrm{a}$ otherwise. Apply Lemma 1 to $t$ and $u$ : the tree $\Delta_{1} \cdot \Delta_{2}^{\omega}$ (using the notations of Lemma 13) is the tree whose nodes are all labelled by a, thus $\Delta_{1} \cdot \Delta_{2}^{\omega} \notin L$, leading to a contradiction.

TheOREm 21. There is a qualitative tree language that is not regular.

Proof. Let $\mathcal{L}_{\mathrm{a}}$ be the language of trees whose set of branches containing at least one a has measure 1. This language is qualitative as noticed in Example 7. In the sequel, we prove that $\mathcal{L}_{\mathrm{a}}$ is not regular.

We first prove that, for any regular tree $t$, if there is no cone in $t$ whose branches only contain the letter $\mathrm{b}$, then $t \in \mathcal{L}_{\mathrm{a}}$. Let $t$ be a regular tree, we can assume w.l.o.g. that if there is a node labelled by a then all its descendants are labeled by a. Then the property "there is no cone in $t$ whose branches only contain $\mathrm{b}$ " is the same as "every subtree contains a subtree made only of a". Let $X_{1}, \ldots, X_{n}$ be the $n$ different subtrees of $t$, and for all $i$, let $\mu_{i}$ be the measure of the set of branches containing a in $X_{i}$ (we call it the value of $X_{i}$ ). We can assume w.l.o.g. that $\forall i \mu_{1} \leqslant \mu_{i}$. If $X_{i_{1}}$ and $X_{i_{2}}$ are the two sons of $X_{1}$, we know that $\mu_{1}=\frac{\mu_{i_{1}}+\mu_{i_{2}}}{2}$. Since $\mu_{1} \leqslant \mu_{i}$ for $i=i_{1}, i_{2}, \mu_{i_{1}}=\mu_{i_{2}}=\mu_{1}$. Hence we can prove by induction that for all $X_{i}$ of minimal value, all the subtrees of $X_{i}$ have minimal value too. Since there is a subtree of a (of value 1 ) in $X_{1}, \mu_{1}=1$ hence for all $i, \mu_{i}=1$, hence the value of $t$ is 1 , hence $t \in \mathcal{L}_{\mathrm{a}}$.

We assume by contradiction that $\mathcal{L}_{\mathrm{a}}$ is regular. The closure properties of regular tree languages implies that the following language $\mathcal{L}$ is also regular:

$$
\mathcal{L}=\left\{t \mid t \notin \mathcal{L}_{\mathrm{a}} \wedge \text { "there is no cone in } t \text { whose branches only contain b" }\right\}
$$

Using our previous characterisation of regular trees in $\mathcal{L}_{\mathrm{a}}$ it follows that $\mathcal{L}$ does not contain any regular tree, hence $\mathcal{L}$ is empty (Theorem 2). Then, to raise a contradiction, we build a (non-regular) tree $t_{0} \in \mathcal{L}$.

For every node $u \in\{0,1\}^{*}$, we let $n_{u}$ be the integer whose binary representation is $1 \cdot u$. We define a tree $t_{0}$ as follows: let $v \in\{0,1\}^{*}$, if there exists some $u$ such 


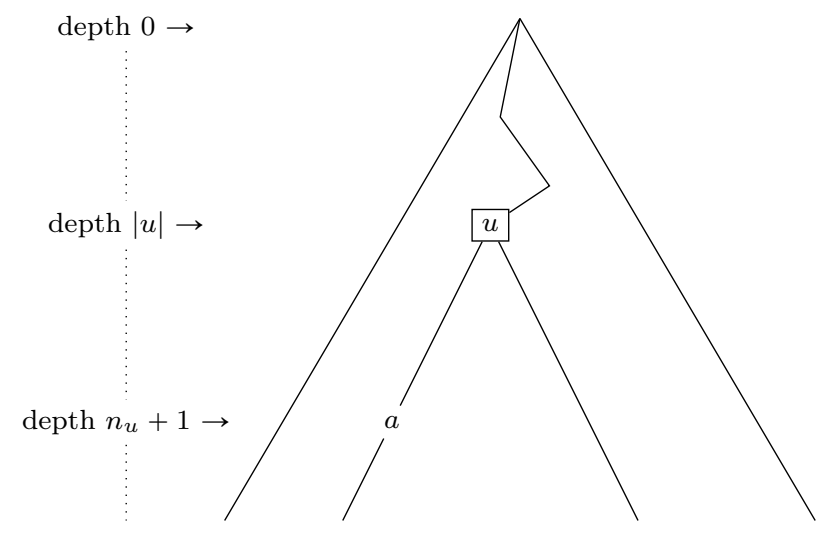

Fig. 4. The tree $t_{0}$

that $v=u .0^{n_{u}+1-|u|}$ then $t_{0}(v)=\mathrm{a}$, otherwise $t_{0}(v)=\mathrm{b}$ (see Figure 4). We now establish that $t_{0} \notin \mathcal{L}_{\mathrm{a}}$. First note that the set of branches in $t_{0}$ that contains at least one a is obtained by taking the union of those cones $\mathcal{C}(u)$ such that $t_{0}(u)=\mathrm{a}$. Then remark that, for every level $\ell$, there is one and only one node $u$ of depth $\ell$ labelled by a (except for $\ell=0,1$ where there are no such $u$ ). Thus we can bound the measure $\mu$ of the set of branches in $t_{0}$ that contains at least one a:

$$
\mu \leqslant \sum_{\ell=2}^{+\infty} 2^{-\ell}=\frac{1}{2}
$$

This proves that $t_{0} \notin \mathcal{L}_{\mathrm{a}}$. Moreover, it follows from the definition, that for every node $u$, there is a branch (the leftmost one) in the cone $\mathcal{C}(u)$ that contains an a, hence $t_{0} \in \mathcal{L}$, which contradicts the fact that $\mathcal{L}$ is empty.

\subsection{The Value of a Tree May not be Reached}

So far we defined qualitative acceptance of a tree by the existence of a run whose set of accepting branches has measure 1 . We can refine this notion by defining the value of a tree as follows. For a tree automaton $\mathcal{A}$, and a tree $t$ we let

$$
\operatorname{Val}_{\mathcal{A}}(t)=\sup _{\rho_{t} \text { run of } \mathcal{A} \text { over } t} \mu\left(\operatorname{AccBr}\left(\rho_{t}\right)\right)
$$

In particular $L_{\text {Qual }}(\mathcal{A})$ is the set of trees $t$ whose value is 1 and is reached for some run (i.e. the sup is a max). The following result proves that the value may not be reached by some run.

THEOREM 22. There is a reachability automaton $\mathcal{A}$ and a tree $t$ such that $\operatorname{Val}_{\mathcal{A}}(t)=$ 1 but $t \notin L_{\text {Qual }}(\mathcal{A})$.

Proof. Let $\mathcal{A}=\left\langle A, Q, q_{w}, \Delta,\left\{q_{a c c}\right\}\right\rangle$ with $A=\{\mathrm{a}, \mathrm{b}\}, Q=\left\{q_{w}, q_{l}, q_{a c c}, q_{r e j}\right\}$, and $\Delta$ contains the following transitions: $q_{w} \stackrel{*}{\rightarrow}\left(q_{w}, q_{w}\right), q_{w} \stackrel{*}{\rightarrow}\left(q_{l}, q_{a c c}\right), q_{l} \stackrel{\text { a }}{\rightarrow}$ $\left(q_{a c c}, q_{l}\right), q_{l} \stackrel{\mathrm{b}}{\rightarrow}\left(q_{r e j}, q_{r e j}\right)$ (here $*$ is a shorthand for an arbitrary letter). Intuitively, the automaton can wait in state $q_{w}$ as long as it wants. It can at some point use the second transition: this leads to accept (all branches in) the subtree rooted at 
the left son, as well as those subtrees rooted in the branch going left and then right forever as long as this branch does not contain a node labelled $b$, in which case the subtree rooted at this node is rejected.

Define $h:\{0,1\}^{*} \rightarrow\{0,1\}^{*}$ by letting $h(u)=u \cdot 0.1^{|u|}$, for all $u \in\{0,1\}^{*}$. Now, consider the tree $t$ defined by $t(u)=\mathrm{b}$ if $u$ belongs to $h\left(\{0,1\}^{*}\right)$ - the image of the set $\{0,1\}^{*}$ by $h-$ and $t(u)=$ a otherwise.

We claim that any run $\rho_{t}$ of $\mathcal{A}$ on $t$ is such that $\mu\left(\operatorname{AccBr}\left(\rho_{t}\right)\right)<1$. Indeed, either the only transition used in $\rho_{t}$ is $q_{w} \stackrel{*}{\rightarrow}\left(q_{w}, q_{w}\right)$ and therefore all branches are

rejecting. Otherwise the transition $q_{w} \stackrel{*}{\rightarrow}\left(q_{l}, q_{a c c}\right)$ is used at least once. Then pick $u$ a node of minimal depth where this transition is used: the cone Cone $(h(u))$ is rejecting and therefore $\mu\left(\operatorname{AccBr}\left(\rho_{t}\right)\right) \leqslant 1-2^{|h(u)|}<1$.

Now, for all integer $i \geqslant 1$, consider the run $\rho_{t}^{i}$ where the transition $q_{w} \stackrel{*}{\rightarrow}\left(q_{w}, q_{w}\right)$ is used for all nodes at depth $<i$, and the transition $q_{w} \stackrel{*}{\rightarrow}\left(q_{l}, q_{a c c}\right)$ is used for all nodes at depth $i$ (then there is no more freedom in defining for the rest of the run). Then for all node $u$ at depth $i$, the branches in Cone $(h(u))$ are rejecting and those in Cone $(u) \backslash$ Cone $(h(u))$ are accepting. Therefore $\mu\left(\operatorname{AccBr}\left(\rho_{t}\right)\right)=1-2^{i} \times 2^{-(i+1+i)}=$ $2^{-(i+1)}$. Thus, $\sup _{\rho_{t}^{i}} \mu\left(\operatorname{AccBr}\left(\rho_{t}\right)\right)=1$, implying that $\operatorname{Val}_{\mathcal{A}}(t)=1$.

Actually, the proof of Theorem 16 directly leads the following.

Corollary 23. Let $\mathcal{A}$ be a parity tree automaton and let $t$ be a tree. Then $\operatorname{Val}_{\mathcal{A}}(t)=\operatorname{Val}\left(\mathbb{G}_{\mathcal{A}, t}\right)$.

\subsection{Positive Tree Languages}

So far, we favoured the almost-sure acceptance condition (i.e. requiring the measure to be equal to 1 ) over the positive one (i.e. requiring the measure to be strictly positive). However, the decidability results on MDP stated in Theorem 3 still hold if we replace the almost-sure acceptance by the positive acceptance [Courcoubetis and Yannakakis 1990; Chatterjee et al. 2004]. We now discuss the impact when considering positive acceptance instead of almost-sure acceptance, and motivate our choice to focus on almost-sure acceptance.

We say that a run $\rho$ of a tree automaton $\mathcal{A}$ is positively accepting if the measure of its set of accepting branches is (strictly) positive, i.e. $\mu(\operatorname{AccBr}(\rho))>0$. A tree $t$ is positively accepted if there exists a positively accepting run of $\mathcal{A}$ over $t$, and we denote by $L_{\mathrm{Pos}}(\mathcal{A})$ the set of all trees positively accepted by $\mathcal{A}$. Finally, a positive tree language is a language $L$ of trees such that there exists a parity automaton $\mathcal{A}$ with $L_{\mathrm{Pos}}(\mathcal{A})=L$.

Note that, contrarily to the qualitative semantics, we can no longer assume that our automata are complete. In particular, the naive idea of adding a sink state does not work, as it would have new runs that may be accepting (going to the sink state yields a rejecting cone, but this may not affect the positivity of the measure of the set of accepting branches).

EXAMPLE 24. Consider the language $\mathcal{L}_{a}^{>0}$ of $\{a, b\}$-labeled trees that have a nonnegligible set of branches containing infinitely many a's. This language is positively accepted by a deterministic Büchi tree automaton (that goes in a final state whenever an a is read and in a non-final state otherwise) and hence is a positive tree language. 
REMARK 25. The complement of a language qualitatively accepted by a deterministic tree automaton $\mathcal{A}$ is positively accepted by a deterministic tree automaton $\mathcal{B}$. Indeed, it suffices to define $\mathcal{B}$ starting from $\mathcal{A}$ and dualise its acceptance condition (namely increment the value of the colouring function by 1 ).

Let us now give two examples of languages that are not positive tree languages. For this we introduce the language $\mathcal{L}_{\mathrm{a} \wedge \mathrm{a}}$ of $\{\mathrm{a}, \mathrm{b}\}$-labelled trees containing an a in both their left and right subtrees that is formally defined as $\mathcal{L}_{\mathrm{a} \wedge \mathrm{a}}=\{t \mid \exists u, v \in$ $\{0,1\}^{*}$ s.t. $\left.t(0 u)=t(1 v)=\mathrm{a}\right\}$.

Proposition 26. The language $\mathcal{L}_{a}$ of $\{a, b\}$-labelled trees whose set of branches containing an a has measure 1 and the language $\mathcal{L}_{a \wedge a}$ of $\{a, b\}$-labelled trees containing an a in both their left and right subtrees are not positive tree languages.

Proof. The proofs for both languages rely on the same family of counterexamples. For every $n \geqslant 0$, let $t_{n}$ be the tree defined by $t_{n}(u)=\mathrm{b}$ for $|u| \leqslant n$ and $t_{n}(u)=\mathrm{a}$ otherwise and let $t_{b}$ be the tree such that $t_{\mathrm{b}}(u)=\mathrm{b}$ for all $u \in\{0,1\}^{*}$.

We are going to show that if $t_{n+2}$ is positively accepted by a parity automaton $\mathcal{A}$ with $n$ states then $\mathcal{A}$ also accepts either the tree $t_{n+2}^{0}$ obtained by replacing the left subtree of $t_{n+2}$ by $t_{\mathrm{b}}$ or the tree $t_{n+2}^{1}$ obtained by replacing the right subtree of $t_{n+2}$ by $t_{b}$.

This property will allow us to conclude as for all $n \geqslant 0, t_{n}$ belongs to $\mathcal{L}_{\mathrm{a}}$ but $t_{n}^{0}$ and $t_{n}^{1}$ do not, $\mathcal{L}_{\mathrm{a}}$ is not a positive language. The same arguments work for $\mathcal{L}_{\mathrm{a} \wedge \mathrm{a}}$.

Let $\rho$ be a positively accepting run of $\mathcal{A}$ on $t_{n+2}$. Without loss of generality, we can assume that the set of accepting branches in the right subtree has a strictly positive measure. We are going to show that $\mathcal{A}$ admits a run $\rho^{\prime}$ (not necessarily a positively accepting one) on $t_{\mathrm{b}}$ starting from $\rho(0)$. By replacing the left subtree of $\rho$ by $\rho^{\prime}$, we then obtain a positively accepting run for $\mathcal{A}$ on $t_{n+2}^{1}$.

For all $i \geqslant 1$, consider the set $Q_{i}=\left\{\rho(0 u) \mid u \in\{0,1\}^{*}\right.$ and $\left.0 \leqslant|u| \leqslant i-1\right\}$ of those states appearing in the left subtree of $\rho$ at depth at most $i$. As the sequence of the $Q_{i}$ is increasing, we have $Q_{n}=Q_{n+1}$. For all $q \in Q_{n}$, there exists a least one transition $\delta_{q}$ of $\mathcal{A}$ of the form $\left(q, \mathrm{~b}, q_{1}, q_{2}\right)$ with $q_{1}$ and $q_{2} \in Q_{n}$ (recall that in $t_{n+2}$ the first $n+2$ levels only contains b's). By using the transition $\delta_{q}$ when in state $q$ and starting from state $q_{0}$, we easily construct a run of $\mathcal{A}$ on $t_{\mathrm{b}}$ starting in state $\rho(0)$.

Proposition 27. The classes of positive and qualitative tree languages are incomparable.

Proof. Let $\mathcal{L}_{\mathrm{a}}$ be the qualitative language of those $\{\mathrm{a}, \mathrm{b}\}$-labelled trees whose set of branches containing an a has measure 1. We proved in Proposition 15 that its complement $\overline{\mathcal{L}_{\mathrm{a}}}$ is not qualitative. But $\overline{\mathcal{L}_{\mathrm{a}}}$ is a positive tree languages, as it is the complement of a language qualitatively accepted by a deterministic tree automaton (see Remark 25).

Conversely we have seen in Proposition 26 the qualitative language $\mathcal{L}_{\mathrm{a}}$ is not positive.

REMARK 28. A natural open question is whether a tree language which is both positively and qualitatively accepted is always regular.

ACM Transactions on Computational Logic, Vol. V, No. N, Month 20YY. 
As it is the case for qualitative languages, the positive languages are incomparable with the regular languages.

Proposition 29. The class of positive tree languages is incomparable with the class of regular languages.

Proof. The language $\mathcal{L}_{\mathrm{a} \wedge \mathrm{a}}$ of $\{\mathrm{a}, \mathrm{b}\}$-labelled trees containing an a in both their left and right subtrees is an example of a regular language that is not positive tree language (cf. Proposition 26).

We are now going to show that the positive tree language $\mathcal{L}_{\mathrm{a}}^{>0}$ of $\{\mathrm{a}, \mathrm{b}\}$-labeled trees that have a non-negligible set of branches containing infinitely many a's (cf. Example 24) is not a regular language.

Toward a contradiction, we assume that $\mathcal{L}_{\mathrm{a}}^{>0}$ is accepted by a parity tree automaton $\mathcal{A}=\left\langle A, Q, q_{i n i}, \Delta, \mathrm{Col}\right\rangle$ (with the standard acceptance condition for runs).

For every $i \geqslant 1$, we let $r_{i}$ be the (finite) tree whose domain consists of all words of length at most $i$ and in which only $0^{i}$ is labeled by a and all other nodes are labelled by $\mathrm{b}$. We denote by $\left|r_{i}\right|=i$ the height of $r_{i}$. The probability $p_{r_{i}}$ to visit an a-labelled node in $r_{i}$ when starting from the root is $\frac{1}{2^{i}}$. We let $\mathcal{T}$ denote the set $\left\{r_{i} \mid i \geqslant 1\right\}$ of all such trees.

To every sequence $s=\left(t_{i}\right)_{i \geqslant 0}$ of trees in $\mathcal{T}$, we associate an infinite $\{\mathrm{a}, \mathrm{b}\}$-labelled tree $t_{s}$ which is intuitively obtained as follows: start with $t_{1}$ and glue to every leaf a copy of $t_{2}$, to every leaf glue a copy of $t_{3}$ and so on. Formally $t_{s}(u)=\mathrm{a}$ if $u$ is of the form $v 0^{\left|t_{i}\right|}$ with $|v|=\sum_{j<i}\left|t_{j}\right|$ and $t_{s}(u)=\mathrm{b}$ otherwise.

By Borel-Cantelli's lemma [Bauer 1996, Lemma 11.1 - p. 70], the tree $t_{s}$ belongs to $L_{\mathrm{a}}^{>0}$ if and only if $\sum_{i \geqslant 0} p_{r_{i}}=+\infty$.

We extend the notion of runs for $\mathcal{A}$ to finite trees. A run of $\mathcal{A}$ on finite tree $t:\{0,1\}^{\leqslant n} \mapsto\{\mathrm{a}, \mathrm{b}\}$ is a finite tree $\rho:\{0,1\}^{\leqslant n} \mapsto Q$ such that for all $u \in\{0,1\}^{<n}$, $(\rho(u), t(a), \rho(u 0), \rho(u 1))$ belongs to $\Delta$. We can summarise the run $\rho$ by a finite information called the profile of the run and denoted $\operatorname{Prof}(\rho)$. The profile $\operatorname{Prof}(\rho)$ of $\rho$ is defined as:

$$
\operatorname{Prof}(\rho)=(\rho(\varepsilon), R) \quad \text { where } \quad R=\left\{(m, \rho(u)) \mid u \in\{0,1\}^{n} \text { and } m=\inf _{v \sqsubseteq u} \operatorname{Col}(v)\right\} .
$$

By extension, the profile of a finite tree $t$, denoted $\operatorname{Prof}(t)$, is the set $\{\operatorname{Prof}(\rho) \mid$ $\rho$ run of $\mathcal{A}$ on $t\}$ of all possible profiles of runs of $\mathcal{A}$ on $t$.

This notion induces an equivalence relation on finite trees denoted $\equiv_{\mathcal{A}}$ which intuitively equates trees that cannot be distinguished by $\mathcal{A}$. Formally for all finite tree $t$ and $t^{\prime}, t \equiv_{\mathcal{A}} t^{\prime}$ if and only if $\operatorname{Prof}(t)=\operatorname{Prof}\left(t^{\prime}\right)$.

The following claim is straightforward.

Claim 30. Let $s=\left(t_{i}\right)_{i \geqslant 1}$ and $s^{\prime}=\left(t_{i}^{\prime}\right)_{i \geqslant 1}$ be two sequences of finite trees in $\mathcal{T}$. If for all $i \geqslant 1, t_{i} \equiv_{\mathcal{A}} t_{i}^{\prime}$ then $\mathcal{A}$ accepts $t_{s}$ if and only if $\mathcal{A}$ accepts $t_{s^{\prime}}$.

Now, note that there are only finitely many different possible profiles hence, there exists an infinite sequence $s=\left(r_{i_{j}}\right)_{j \geqslant 0}$ of trees with the same profile and such that the sequence $\left(i_{j}\right)_{j \geqslant 0}$ is strictly increasing. As the $\left(i_{j}\right)_{j \geqslant 0}$ sequence is strictly increasing it implies that $\sum_{j \geqslant 0} p_{r_{i_{j}}}<+\infty$, hence the tree $t_{s}$ does not belong to $L_{\mathrm{a}}^{>0}$. Now consider the constant sequence $s^{\prime}=r_{i_{0}}, r_{i_{0}}, \ldots$ one has $\sum_{j \geqslant 0} p_{r_{i_{0}}}=+\infty$ 
hence $t_{s^{\prime}}$ belongs to $L_{\mathrm{a}}^{>0}$. This leads a contradiction with $\mathcal{A}$ accepting $L_{\mathrm{a}}^{>0}$ as $\mathcal{A}$ behaves the same on both $t_{s}$ and $t_{s^{\prime}}$,i.e. $\mathcal{A}$ accepts $t_{s}$ if and only if $\mathcal{A}$ accepts $t_{s^{\prime}}$.

The most notable difference between positive and qualitative tree languages is that positive tree languages are not closed under intersection.

Proposition 31. The class of positive tree languages is closed under union but neither under intersection nor complementation.

Proof. The closure under union is immediate thanks to non-determinism. The non-closure under complementation will be a consequence of the closure under union and the non closure under intersection. However, one can also derive it directly by considering the language $\mathcal{L}_{\mathrm{a}}$ : we have shown in the proof of Proposition 26 that this language is not positive while its complement is positive.

We now prove the non-closure under intersection. Consider the language $L_{0}$ (resp. $L_{1}$ ) of those trees $t$ containing a non-negligible set of branches containing an $a$ in the left (resp. right) subtree of the root. Both languages are positively accepted by a deterministic reachability automaton. But we have seen in Proposition 26 that their intersection $\mathcal{L}_{\mathrm{a} \wedge \mathrm{a}}$ is not a positive tree language.

However, positive tree languages enjoy all decidability properties of qualitative languages presented in Section 3.5.

TheOREM 32. The tree $t$ belongs to $L_{\mathrm{Pos}}(\mathcal{A})$ if and only if Éloïse positively wins in $\mathbb{G}_{\mathcal{A}, t}$.

Proof. The proof is identical to that of Theorem 16.

THEOREM 33. The language $L_{\mathrm{Pos}}(\mathcal{A})$ is non empty if and only if Élö̈se positively wins in $\mathbb{G}_{\mathcal{A}}$ from $q_{\mathrm{ini}}$.

Proof. The proof is identical to that of Theorem 17.

Corollary 34. Let $\mathcal{A}$ be a parity tree automaton. Then one can decide whether $L_{\mathrm{Pos}}(\mathcal{A})=\varnothing$ in polynomial time. Moreover, if $L_{\mathrm{Pos}}(\mathcal{A}) \neq \varnothing$, it contains a regular tree, and such a tree can be constructed in polynomial time.

Proof. As the properties expressed in Theorem 3 of MDP with almost-sure winning condition also hold for the positive winning condition, we can use the same proof as for Corollary 18.

\section{BEYOND NON-DETERMINISTIC AUTOMATA: THE PROBABILISTIC SETTING}

Following [Rabin 1963] for finite words and [Baier and Größer 2005; Baier et al. 2008; Baier et al. 2012] for infinite words we investigate probabilistic automata on infinite trees. That is the set of transitions of an automaton is replaced by a probability distribution over the set of all transitions which induces a probability measure on the set of runs of the automaton. Now, a tree is accepted if almost every run over the input tree is accepting. For the run, we may use either the classical or the qualitative acceptance criterion.

ACM Transactions on Computational Logic, Vol. V, No. N, Month 20YY. 


\subsection{Definitions}

4.1.1 Probabilistic Tree Automata. A probabilistic tree automaton $\mathcal{A}$ is a tuple $\left\langle A, Q, q_{i n i}, \delta\right.$, Acc $\rangle$ where $A$ is the input alphabet, $Q$ is a finite set of states, $q_{\mathrm{ini}} \in Q$ is the initial state, Acc $\subseteq Q^{\omega}$ is the acceptance condition and $\delta$ is a mapping from $Q \times A \times Q \times Q$ to [0,1] such that for all $q \in Q$ and a $\in A$, $\sum_{q_{0}, q_{1} \in Q} \delta\left(q, \mathrm{a}, q_{0}, q_{1}\right)=1$. Intuitively, the value $\delta\left(q, \mathrm{a}, q_{0}, q_{1}\right)$ is the probability for a transition $q \stackrel{\text { a }}{\rightarrow}\left(q_{0}, q_{1}\right)$ to be used by the automaton when it is in state $q$ and reads the symbol a.

This probability distribution on the transitions induces a probability measure on the set of runs of $\mathcal{A}$. In this setting, a run of $\mathcal{A}$ is simply a $Q$-labeled tree whose root is labeled by the initial state $q_{\text {ini }}$. We denote by $\operatorname{Runs}(\mathcal{A})$ (or simply Runs if $\mathcal{A}$ is clear from the context) the set of all runs of $\mathcal{A}$. We denote by $\operatorname{AccRuns}(\mathcal{A})$ the set of accepting runs of $\mathcal{A}$ and by $\operatorname{QualAccRuns}(\mathcal{A})$ the set of qualitatively accepting runs of $\mathcal{A}$.

In the sequel, we will show that, for a given tree $t$ the sets $\operatorname{AccRuns}(\mathcal{A})$ and QualAccRuns $(\mathcal{A})$ are measurable (Proposition 38), and this will allows us to define almost-sure acceptance of a tree by a probabilistic automaton.

4.1.2 Measurability of $\operatorname{AccRuns}(\mathcal{A})$ and $\operatorname{Qual} \operatorname{AccRuns}(\mathcal{A})$. We first define a $\sigma$ algebra for $\operatorname{Runs}(\mathcal{A})$. A partial run is a partial function $\lambda:\{0,1\}^{*} \rightarrow Q$ with $\lambda(\varepsilon)=q_{\text {ini }}$ and such that $\operatorname{Dom}(\lambda)$ is finite, non-empty, prefix-closed and proper, i.e. such that for all $w \in\{0,1\}^{*}, w 0 \in \operatorname{Dom}(\lambda)$ iff $w 1 \in \operatorname{Dom}(\lambda)$. If $\lambda$ is a partial run, we denote by $\operatorname{Inner}(\lambda)$ the set $\{w \in \operatorname{Dom}(\lambda) \mid w 0 \in \operatorname{Dom}(\lambda)$ and $w 1 \in \operatorname{Dom}(\lambda)\}$ of non-leaf nodes. The cylinder associated to $\lambda$, denoted $\operatorname{Cyl}(\lambda)$, is the set of runs consistent with $\lambda$, i.e.

$$
\operatorname{Cyl}(\lambda)=\{\rho \in \operatorname{Runs} \mid \forall w \in \operatorname{Dom}(\lambda), \rho(w)=\lambda(w)\}
$$

Let $\mathcal{F}_{R}$ be the $\sigma$-algebra generated by the cylinders. By Carathéodory's extension theorem, there exists a unique probability measure $\mu_{t}$ on the measurable space (Runs, $\mathcal{F}_{R}$ ) such for all partial run $\lambda:\{0,1\}^{*} \rightarrow Q$,

$$
\mu_{t}\left(\mathrm{Cyl}_{\mathcal{A}}(\lambda)\right)=\prod_{w \in \operatorname{Inner}(\lambda)} \delta(\lambda(w), t(w), \lambda(w 0), \lambda(w 1))
$$

Note that both $\mu_{t}$ and (Runs, $\mathcal{F}_{R}$ ) depend on $t$. Also note that the definition of $\mu_{t}$ does not make sense without the properness condition in the definition of a partial run.

REMARK 35. A balanced partial run $\lambda$ is a mapping from $\{0,1\}^{n}$ to $Q$ for some $n \geqslant 0$ with $\lambda(\varepsilon)=q_{\mathrm{ini}}$. Hence it is a special kind of partial play in the previous sense. It is straightforward to note that the $\sigma$-algebra generated by balanced partial play is $\mathcal{F}_{R}$. Moreover one has

$$
\mu_{t}\left(\mathrm{Cyl}_{\mathcal{A}}(\lambda)\right)=\prod_{w \in\{0,1\} \leqslant n-1} \delta(\lambda(w), t(w), \lambda(w 0), \lambda(w 1))
$$

Let $\mathcal{A}$ be an automaton and define the mapping $f_{\mathcal{A}}: \operatorname{Runs} \times \operatorname{Br} \rightarrow[0,1]$ associating to a pair $(\rho, \pi) \in$ Runs $\times$ Br the value 1 if $\rho(\pi)$ belongs to Acc and 0 otherwise. The following lemma proves that $f_{\mathcal{A}}$ is integrable. 
LEMMA 36. Let $\mathcal{A}$ be a probabilistic tree automaton with an $\omega$-regular acceptance condition and let $t$ be a tree. The mapping $f_{\mathcal{A}}$ is integrable in the product space $\left(\operatorname{Runs}(\mathcal{A}), \mathcal{F}_{R}, \mu_{t}\right) \otimes\left(\mathrm{Br}, \mathcal{F}_{\mathrm{Br}}, \mu\right)$.

Proof. The product space $\left(\mathrm{Runs}, \mathcal{F}_{R}\right) \otimes\left(\mathrm{Br}, \mathcal{F}_{\mathrm{Br}}\right)$ is generated by the sets $\operatorname{Cone}(w) \times \operatorname{Cyl}(\lambda)$ for all $w \in\{0,1\}^{*}$ and all partial run $\lambda$ (cf. [Bauer 2001, Theorem 22.1 - p. 132]). Thanks to Remark 35 we can restrict our attention to balanced partial runs.

According to [Bauer 2001, Theorem 9.1 - p. 50], it is enough to show that $\operatorname{AccPairs}(\mathcal{A})=\{(\rho, \pi) \mid \rho(\pi) \in \operatorname{Acc}\}$ is measurable in the product space.

We first establish the result for a Büchi acceptance condition. For that, we let $\mathcal{A}=\left\langle A, Q, q_{\text {ini }}, \delta, F\right\rangle$ be probabilistic tree automaton with a Büchi acceptance condition.

For $u \in\{0,1\}^{*}$, we introduce the set $R_{u}$ of all pairs $(\rho, \pi) \in \operatorname{Runs} \times \operatorname{Br}$ such that $\pi$ admits $u$ as a prefix and $\rho(v) \notin F$ for all $u \sqsubseteq v \sqsubseteq \pi$. The set $\operatorname{AccPairs}(\mathcal{A})$ is the complement of the countable union $\bigcup_{u \in\{0,1\} *} R_{u}$. Hence, it is enough to show $R_{u}$ is measurable for all $u \in\{0,1\}^{*}$.

Let us fix a node $u \in\{0,1\}^{*}$. For all $n>|u|$, let $C_{n}$ be the union all sets $\operatorname{Cyl}(\lambda) \times \operatorname{Cone}(w)$ where $\lambda:\{0,1\}^{n} \rightarrow Q$ is a partial run and $w \in\{0,1\}^{n} \supsetneqq u$ is a word such that $\lambda(v) \notin F$ for all $u \sqsubseteq v \sqsubseteq w$. We claim that the set $R_{u}$ is equal to $\bigcap_{n>|u|} C_{n}$ and hence is measurable. The direct inclusion, $R_{u} \subseteq \bigcap_{n>|u|} C_{n}$, is obvious. For the converse one, let $(\rho, \pi)$ be in $\bigcap_{n>|u|} C_{n}$. For all $n>|u|$, the node $w_{n} \in\{0,1\}^{n}$ witnessing that $(\rho, \pi)$ belongs to $C_{n}$ is indeed the unique node of $\pi$ of depth $n$. As $n$ can be arbitrary large, it follows that every node $v$ in $\pi$ of depth $>|u|$ is such $\rho(v) \notin F$, which exactly means that $(\rho, \pi) \in R_{u}$.

The case of the parity conditions follows from the previous case as one can express them as a Boolean combination of Büchi conditions.

The general case of an arbitrary $\omega$-regular condition is obtained as follows. First, one considers a deterministic parity word automaton recognising Acc, and then takes the synchronised product of this automaton with the tree automaton. This leads to a parity tree automaton accepting the same language and whose runs are in bijection with the ones of the original automaton. Moreover as this bijection preserves the set of accepting branches, one can conclude from the parity case.

Note that the previous lemma implies that the set of accepting branches in a run of a tree automaton with an $\omega$-regular acceptance condition is measurable in $\left(\mathrm{Br}, \mathcal{F}_{\mathrm{Br}}\right)$. This leads an alternative proof of Proposition 6.

Corollary 37. Let $\mathcal{A}$ be a tree automaton equipped with an $\omega$-regular acceptance condition, and let $\rho$ be a run of $\mathcal{A}$ over some tree $t$. Then the set $\operatorname{AccBr}(\rho)$ of accepting branches in $t$ is measurable.

Proof. Let $\mathcal{A}$ be a tree automaton with an $\omega$-regular acceptance condition, $t$ be a tree and $\rho$ be a run of $\mathcal{A}$ on $t$. The set $\operatorname{AccBr}(\rho)$ is equal to $\{\lambda \in \operatorname{Br} \mid \rho(\lambda) \in \operatorname{Acc}\}$. As by the proof of Lemma $36, \operatorname{AccPairs}(\mathcal{A})$ is measurable in the product space, $\operatorname{AccBr}(\rho)$ is also measurable (cf. [Bauer 2001, Lemma 23.1 - p. 135]).

We are now ready to establish the measurability of the sets $\operatorname{AccRuns}(\mathcal{A})$ and $\operatorname{QualAccRuns}(\mathcal{A})$.

ACM Transactions on Computational Logic, Vol. V, No. N, Month 20YY. 
Proposition 38. For all probabilistic tree automata $\mathcal{A}$ with an $\omega$-regular acceptance condition, the sets $\operatorname{AccRuns}(\mathcal{A})$ and $\mathrm{Qual} \operatorname{AccRuns}(\mathcal{A})$ are measurable.

Proof. We first consider the case of $\operatorname{AccRuns}(\mathcal{A})$. As seen in Proposition 6, it is enough to establish the property for the Büchi acceptance condition.

Let $\left\langle A, Q, q_{i n i}, \delta, F\right\rangle$ be probabilistic tree automaton with a Büchi acceptance condition.

For $u \in\{0,1\}^{*}$, we introduce the set $R_{u}$ of all runs $\rho$ of $\mathcal{A}$ for which there exists an infinite branch $\pi$ such that $u \sqsubseteq \pi$ and for all $u \sqsubseteq v \sqsubseteq \pi, \rho(v) \notin F$. The set of accepting runs $\operatorname{AccRuns}(\mathcal{A})$ is the complement of the countable union $\bigcup_{u \in\{0,1\} *} R_{u}$. Hence it is enough to show $R_{u}$ is measurable for all $u \in\{0,1\}^{*}$.

Let us fix a node $u \in\{0,1\}^{*}$. For all $n>|u|$, let $C_{n}$ be the union of all cylinders $\operatorname{Cyl}(\lambda)$ where $\lambda:\{0,1\}^{n} \rightarrow Q$ is a (balanced) partial run for which there exists a word $w \in\{0,1\}^{n} \sqsupseteq u$ such that $\lambda(v) \notin F$ for all $u \sqsubseteq v \sqsubseteq w$.

We claim that $R_{u}$ is equal to $\bigcap_{n>|u|} C_{n}$ and is hence measurable. The direct inclusion, $R_{u} \subseteq \bigcap_{n>|u|} C_{n}$, is obvious. For the converse one, let $\rho$ be a run in $\bigcap_{n>|u|} C_{n}$. For all $n>|u|$, let $w_{n}$ be a word in $\{0,1\}^{n}$ witnessing that $\rho$ belongs to $C_{n}$. Let $T$ be the prefix-closure of the set $\left\{w_{n}|n>| u \mid\right\}$. By Koenig's lemma, $T$ contain an infinite branch $\pi$. Clearly $u$ is a prefix of $\pi$ and for all $u \sqsubseteq v \sqsubseteq \pi$, $\rho(v) \notin F$ as $v$ is a prefix of one of the $w_{n}$. Hence $\rho$ belongs to $R_{u}$.

Let us now consider the case of $\operatorname{QualAccRuns}(\mathcal{A})$. From Lemma 36 we have that $\operatorname{AccPairs}(\mathcal{A})=\{(\rho, \pi) \mid \rho(\pi) \in \operatorname{Acc}\}=f_{\mathcal{A}}^{-1}(\{1\})$ is measurable in the product space. Hence the numerical function $g:$ Runs $\rightarrow[0,1]$ associating to a run $\rho$ the measure of its set of accepting branches $\left(\right.$ i.e. $\mu\left(\operatorname{AccPairs}(\mathcal{A})_{\rho}\right)$ where $\operatorname{AccPairs}(\mathcal{A})_{\rho}=\{\lambda \in$ $\operatorname{Br} \mid \rho(\lambda) \in \mathrm{Acc}\}$ ) is measurable (cf. [Bauer 2001, Lemma 23.1 - p. 135]). As QualAccRuns $(\mathcal{A})$ is equal to $g^{-1}(\{1\})$, it is measurable.

4.1.3 Almost-Surely Accepted Trees. Proposition 38 shows that the following definition is well-founded.

A tree $t$ is (almost-surely) accepted by $\mathcal{A}$ with the classical (resp. qualitative) semantics if almost all runs of $\mathcal{A}$ on $t$ are accepting (resp. qualitatively accepting), i.e. $\mu_{t}(\operatorname{AccRuns}(\mathcal{A}))=1\left(\operatorname{resp} . \mu_{t}(\operatorname{QualAccRuns}(\mathcal{A}))=1\right)$. We denote by $L^{=1}(\mathcal{A})$ (resp. $\left.L_{\mathrm{Qual}}^{=1}(\mathcal{A})\right)$ the set of trees accepted by $\mathcal{A}$ with the classical (resp. qualitative) semantics for runs. More formally, we define $L^{=1}(\mathcal{A})=\left\{t \mid \mu_{t}(\operatorname{AccRuns}(\mathcal{A}))=1\right\}$ and $L_{\text {Qual }}^{=1}(\mathcal{A})=\left\{t \mid \mu_{t}(\operatorname{QualAccRuns}(\mathcal{A}))=1\right\}$.

REMARK 39. Our motivation for considering almost-sure acceptation and not positive acceptation is discussed in Section 4.5.

The following easy lemma is used later to prove Proposition 42 and Proposition 43.

LEMMA 40. Let $(\Omega, \mathcal{F}, \mu)$ be a probability space and $f$ be a measurable function from $\Omega$ to $[0,1]$ then $\int_{\Omega} f d \mu=1$ if and only if $\mu\left(f^{-1}(\{1\})\right)=1$.

Proof. As $f$ is bounded, measurability implies integrability. Consider the integrable mapping $g=1-f$. Clearly $\int_{\Omega} f d \mu=1$ if and only if $\int_{\Omega} g d \mu=0$. By [Bauer 2001, Theorem 13.2 - p. 71], $\int_{\Omega} g d \mu=0$ if and only if $g^{-1}(\{0\})$ has measure 1 . The announced equivalence follows. 
REMARK 41. The definition of [Baier and Größer 2005] (for $\omega$-words) allows for incomplete automata (i.e. for all $q \in Q$, the sum $\sum_{q^{\prime} \in Q} \delta\left(q, a, q^{\prime}\right)=1$ or 0 ). However it is easy to verify that every automaton can be rendered complete without changing the acceptance condition and the measure of acceptation. Remark that removing all states $q$ such that $\sum_{q_{1}, q_{2} \in Q} \delta\left(q, a, q_{1}, q_{2}\right)=0$ does not change the measure of the set of accepting runs (either in the classical or qualitative sense). By iteratively applying this process, we obtain an equivalent complete automata.

Combining Lemma 36 and Lemma 40, we can show that the almost-sure acceptation of a tree $t$ by an automaton $\mathcal{A}$ for the qualitative semantics can be defined by integrating the mapping $f_{\mathcal{A}}$.

Proposition 42. Let $\mathcal{A}$ be a probabilistic tree automaton with an $\omega$-regular acceptance condition and let $t$ be a tree. Then we have:

$$
t \in L_{\text {Qual }}^{=1}(\mathcal{A}) \Leftrightarrow \int f_{\mathcal{A}} d \mu_{t} \otimes \mu=1
$$

Proof. As $f_{\mathcal{A}}$ is measurable (Lemma 36), by Tonelli's theorem [Bauer 2001, Theorem 23.6 - p. 138], the mapping $g:$ Runs $\rightarrow[0,1]$ associating to a run $\rho \in$ Runs the value $\int_{\mathrm{Br}} f_{\mathcal{A}}(\rho, \cdot) d \mu$ is measurable.

$$
\begin{array}{lll} 
& t \text { belongs to } L_{\mathrm{Qual}}^{=1}(\mathcal{A}) & \\
\text { iff } & \mu_{t}\left(g^{-1}(\{1\})\right)=1 & \\
\text { iff } & \int_{\operatorname{Runs}} g d \mu_{t}=1 & \text { by Lemma } 40 \\
\text { iff } & \int_{\operatorname{Runs}} \int_{\mathrm{Br}} f_{\mathcal{A}} d \mu d \mu_{t}=1 & \text { by definition of } g \\
\text { iff } & \int_{\mathrm{Runs} \times \operatorname{Br}} f_{\mathcal{A}} d \mu_{t} \otimes \mu=1 & \text { by Tonelli's theorem } \\
& & \text { [Bauer 2001, Theorem 23.6 - p. 138] }
\end{array}
$$

4.1.4 Examples. We conclude this section with examples of languages accepted by probabilistic tree automata.

For an $\omega$-word language $L \subseteq\{\mathrm{a}, \mathrm{b}\}^{\omega}$, we denote by $\mathrm{Path}^{=1}(L)$ the set of trees labeled by $\{\mathrm{a}, \mathrm{b}\}$ with almost all their branch labels in $L$ (i.e. $\mu(\{\pi \in \mathrm{Br} \mid t(\pi) \in$ $L\})=1$ ). It is easy to see that, for any $\omega$-regular language $L$, the tree language $\mathrm{Path}^{=1}(L)$ is a qualitative tree language.

More interestingly, if $L$ is almost-surely accepted by a probabilistic $\omega$-word automaton ${ }^{6}$ with an $\omega$-regular acceptance condition, we can show that $\operatorname{Path}^{=1}(L)$ is accepted by a probabilistic tree automaton (with the qualitative semantics).

Proposition 43. Given a probabilistic $\omega$-word automaton $\mathcal{B}$ with an $\omega$-regular acceptance condition, there exists a probabilistic tree automaton $\mathcal{A}$ with the same acceptance condition such that $L_{\text {Qual }}^{=1}(\mathcal{A})$ is equal to $\operatorname{Path}^{=1}\left(L^{=1}(\mathcal{B})\right)$.

Proof. Let $\mathcal{B}=\left\langle A, Q, q_{\text {ini }}, \delta\right.$, Acc $\rangle$ be a (complete) probabilistic $\omega$-word automaton with an $\omega$-regular condition. Consider the probabilistic tree automaton $\mathcal{A}$

${ }^{6}$ In the context of this article, probabilistic $\omega$-word automata are simply probabilistic tree automata running over unary trees. For such an automaton $\mathcal{B}$, we denote by $L^{=1}(\mathcal{B})$ the language almost-surely accepted by $\mathcal{B}$.

ACM Transactions on Computational Logic, Vol. V, No. N, Month 20YY. 
simulating $\mathcal{B}$ on all branches. Formally $\mathcal{A}$ is equal to $\left\langle A, Q, q_{\text {ini }}, \delta^{\prime}, F\right\rangle$ where, for all $p, q \in Q$ and $x \in A, \delta^{\prime}(p, x, q, q)=\delta(p, x, q)$.

Let $t$ be a tree and let $g: \mathrm{Br} \mapsto[0,1]$ be the mapping associating to a branch $\pi$ the value $\int f_{\mathcal{A}}(\cdot, \pi) d \mu_{t}$. We have the following claim.

Claim 44. For any $\pi \in \mathrm{Br}, g(\pi)=1$ if and only if $t(\pi)$ belongs to $L^{=1}(\mathcal{B})$.

Proof of Claim 44. Let $\pi=a_{1} a_{2} \cdots \in \mathrm{Br}$ and let $w=t(\pi)$. It is enough to show that: $g(\pi)=\int f_{\mathcal{A}}(\cdot, \pi) d \mu_{t}$ is equal to $\mu_{w}(\operatorname{AccRuns}(\mathcal{B}))$. By definition $\int f_{\mathcal{A}}(\cdot, \pi) d \mu_{t}$ is equal to $\mu_{t}\left(\operatorname{AccRuns}_{\pi}(\mathcal{A})\right)$ where $\mu_{t}\left(\operatorname{AccRuns}_{\pi}(\mathcal{A})\right)$ is the set of runs $\rho$ of $\mathcal{A}$ such that $\rho(\pi)$ is accepting.

Consider the mapping $\psi: \operatorname{Runs}(\mathcal{A}) \rightarrow \operatorname{Runs}(\mathcal{B})$ associating to a run $\rho$ of $\mathcal{A}$ the corresponding run $\rho(\varepsilon) \rho\left(a_{1}\right) \cdots$ of $\mathcal{B}$. We claim that $\psi$ is measurable and that $\mu_{w}$ is the image of $\mu_{t}$ under $\psi$. This claim in particular implies that:

$$
\begin{aligned}
\mu_{w}(\operatorname{AccRuns}(\mathcal{B})) & =\mu_{t}\left(\psi^{-1}(\operatorname{AccRuns}(\mathcal{B}))\right. \\
& =\mu_{t}\left(\operatorname{AccRuns}_{\pi}(\mathcal{A})\right) \\
& =\int f_{\mathcal{A}}(\cdot, \pi) d \mu_{t}
\end{aligned}
$$

To substantiate our claim, it is enough to show that $\psi^{-1}(\mathrm{Cyl}(\lambda))$ is a measurable subset of $\operatorname{Runs}(\mathcal{A})$ such that $\mu_{t}\left(\psi^{-1}(\operatorname{Cyl}(\lambda))\right)=\mu_{w}(\operatorname{Cyl}(\lambda))$. The first part implies that $\psi$ is measurable and the second that the image measure of $\mu_{t}$ under $\psi$, denoted $\psi\left(\mu_{t}\right)$, coincide with $\mu_{w}$ on the cylinders. By Carathéodory's unique extension theorem, this in turn implies that $\mu_{w}$ and $\psi\left(\mu_{t}\right)$ coincide.

Let $\lambda=q_{0} \ldots q_{n}$ be a partial run of $\mathcal{B}$. Consider the partial balanced run $\eta$ of $\mathcal{A}$ defined for $i \in[0, n-1]$ by $\eta\left(a_{1} \cdots a_{i-1} 0\right)=\eta\left(a_{1} \cdots a_{i-1} 1\right)=q_{i}$. It is clear that $\psi^{-1}(\operatorname{Cyl}(\lambda))=\operatorname{Cyl}(\eta)$. Moreover we have:

$$
\begin{aligned}
\mu_{t}(\operatorname{Cyl}(\eta)) & =\prod_{j \in[0, n-1]} \delta^{\prime}\left(q_{j}, t\left(a_{1} \cdots a_{j}\right), q_{j+1}, q_{j+1}\right) \\
& =\prod_{j \in[0, n-1]} \delta\left(q_{j}, t\left(a_{1} \cdots a_{j}\right), q_{j+1}\right) \\
& =\mu_{w}(\operatorname{Cyl}(\lambda))
\end{aligned}
$$

Proof of Claim 44

We are now ready to conclude:

$$
\begin{aligned}
& t \in L_{\text {Qual }}^{=1}(\mathcal{A}) \\
& \Leftrightarrow \int f_{\mathcal{A}} d \mu_{t} \otimes \mu=1 \quad \text { Proposition } 42 \\
& \Leftrightarrow \int g d \mu=1 \quad \text { Tonelli's Theorem } \\
& \Leftrightarrow g(\pi)=1 \text { almost everywhere Lemma } 40 \\
& \Leftrightarrow \mu\left(\left\{\pi \mid t(\pi) \in L^{=1}(\mathcal{B})\right\}\right)=1 \quad \text { Claim } 44 \\
& \Leftrightarrow t \in \operatorname{Path}^{=1}\left(L^{=1}(\mathcal{B})\right)
\end{aligned}
$$

\subsection{Acceptance Game for Qualitative Probabilistic Tree Automata}

Fix a probabilistic tree automaton $\mathcal{A}=\left\langle A, Q, q_{\text {ini }}, \delta\right.$, Acc $\rangle$ and a tree $t$. We define an infinite Markov chain $\mathcal{M}_{\mathcal{A}, t}=\left(G_{\mathcal{A}, t}, \mathcal{O}_{\text {Acc }}\right)$ such that $\mathcal{M}_{\mathcal{A}, t}$ almost-surely fulfils its objective iff $t$ belongs to $L_{\text {Qual }}^{=1}(\mathcal{A})$. Compared with the acceptance game for qualitative tree automata, the transition is no longer chosen by Éloïse: it is now randomly chosen with the probability distribution given by $\mathcal{A}$. Hence, this is why we simply obtain a Markov chain instead of an MDP. 
The arena $G_{\mathcal{A}, t}$ is equal to $\left\langle S, s_{\text {ini }}, \zeta\right\rangle$ where $S=Q \times\{0,1\}^{*} \cup \Delta \times\{0,1\}^{*}$ with $\Delta=Q \times Q \times Q, s_{\text {ini }}=\left(q_{\text {ini }}, \varepsilon\right)$ and $\zeta: S \mapsto D(S)$ is defined as follows. For all $w \in$ $\{0,1\}^{*}$ and all $q \in Q, \zeta((q, w))\left(\left(q, q_{0}, q_{1}\right)\right)=\delta\left(q, t(w), q_{0}, q_{1}\right)$ for all $q_{0}, q_{1} \in Q$ and is equal to 0 otherwise. For all $w \in\{0,1\}^{*}$ and $q_{0}, q_{1} \in Q, \zeta\left(\left(\left(q, q_{0}, q_{1}\right), w\right),\left(q_{0}, w 0\right)=\right.$ $\zeta\left(\left(\left(q, q_{0}, q_{1}\right), w\right),\left(q_{1}, w 1\right)\right)=\frac{1}{2}$ and 0 otherwise. Recall that $\mu_{\mathcal{M}_{\mathcal{A}, t}}$ denotes the probability measure associated to $\mathcal{M}_{\mathcal{A}, t}$.

Note that a trace in $\mathcal{M}_{\mathcal{A}, t}$ can be uniquely represented by an infinite sequence $\left(\left(p_{0}, q_{0}^{0}, q_{0}^{1}\right), a_{0}\right)\left(\left(p_{1}, q_{1}^{0}, q_{1}^{0}\right), a_{1}\right) \ldots$ labeled by $\Delta \times\{0,1\}$ such that $p_{0}=q_{\text {ini }}$ and for all $i \geqslant 0, p_{i+1}=q_{i}^{a_{i}}$. The objective $\mathcal{O}_{\text {Acc }}$ is defined as the set of those traces $\left(\left(p_{0}, q_{0}^{0}, q_{0}^{1}\right), a_{0}\right)\left(\left(p_{1}, q_{1}^{0}, q_{1}^{0}\right), a_{1}\right) \ldots$ such that $p_{0} p_{1} \ldots$ belongs to Acc.

Proposition 45. Let $\mathcal{A}$ be a probabilistic tree automaton with an $\omega$-regular acceptance condition and let $t$ be a tree. We have $t \in L_{\mathrm{Qual}}^{=1}(\mathcal{A})$ iff $\mathcal{M}_{\mathcal{A}, t}$ almost-surely fulfils its objective.

Proof. Let AccPairs $(\mathcal{A})=\{(\rho, \pi) \mid \rho(\pi) \in \operatorname{Acc}\} \subseteq$ Runs $\times$ Br. By Proposition 42 , to establish the desired equivalence, it is sufficient to show that $\mu_{t} \otimes$ $\mu(\operatorname{AccPairs}(\mathcal{A}))=\mu_{\mathcal{M}_{\mathcal{A}, t}}\left(\mathcal{O}_{\mathrm{Acc}}\right)$.

Consider the mapping $\psi$ : Runs $\times \mathrm{Br} \mapsto$ Traces associating with a pair $\left(\rho, a_{0} a_{1} \ldots\right)$ the trace $(\rho(\varepsilon), \rho(0), \rho(1)) a_{0}\left(\rho\left(a_{0}\right), \rho\left(a_{0} 0\right), \rho\left(a_{0} 1\right)\right) a_{1} \ldots$ of the Markov chain. It is clear that $\operatorname{AccPairs}(\mathcal{A})=\psi^{-1}\left(\mathcal{O}_{\text {Acc }}\right)$.

We claim that $\psi$ is measurable and that $\mu_{\mathcal{M}_{\mathcal{A}, t}}$ is the image of $\mu_{t} \otimes \mu$ under $\psi$. In particular this implies that $\mu_{\mathcal{M}_{\mathcal{A}, t}}\left(\mathcal{O}_{\mathrm{Acc}}\right)=\mu_{t} \otimes \mu(\operatorname{AccPairs}(\mathcal{A}))$.

To substantiate our claim, it is enough to show, for any partial trace $\theta$, that $\psi^{-1}(\operatorname{Cyl}(\theta))$ is a measurable subset of Runs $\times \operatorname{Br}$ such that $\mu_{t} \otimes \mu\left(\psi^{-1}(\operatorname{Cyl}(\theta))\right)=$ $\mu_{\mathcal{M}_{\mathcal{A}, t}}(\mathrm{Cyl}(\theta))$. The first part implies that $\psi$ is measurable and the second that the image measure of $\mu_{t} \otimes \mu$ under $\psi$, denoted $\psi\left(\mu_{t} \otimes \mu\right)$, coincide with $\mu_{\mathcal{M}_{\mathcal{A}, t}}$ on the cylinders. By Carathéodory's unique extension theorem, this in turn implies that $\mu_{\mathcal{M}_{\mathcal{A}, t}}$ and $\psi\left(\mu_{t} \otimes \mu\right)$ coincide.

Let $\theta=\left(p_{0}, q_{0}^{0}, q_{0}^{1}\right) a_{0} \ldots a_{n-1}\left(p_{n}, q_{n}^{0}, q_{n}^{1}\right)$ be a partial trace. Take $w=a_{0} \ldots a_{n-1}$ and $\lambda$ the (non balanced) partial run of $\mathcal{A}$ defined for all $i \in[0, n]$ by $\lambda\left(a_{0} \cdots a_{i-1}\right)=$ $p_{i}, \lambda\left(a_{0} \cdots a_{i-1} 0\right)=q_{i}^{0}$ and $\lambda\left(a_{0} \ldots a_{i-1} 1\right)=q_{i}^{1}$. We have $\psi^{-1}(\operatorname{Cyl}(\theta))=\operatorname{Cyl}(\lambda) \times$ $\operatorname{Cyl}(w)$. By definition $\mu_{t}(\operatorname{Cyl}(\lambda))$ is equal to $\prod_{0 \leqslant i \leqslant n-1} \delta\left(p_{i}, t\left(a_{0} \cdots a_{i-1}\right), q_{i}^{0}, q_{i}^{1}\right)$ and hence

$$
\begin{aligned}
& \mu_{t} \otimes \mu\left(\psi^{-1}(\operatorname{Cyl}(\theta))\right)=\frac{1}{2^{n}} \prod_{0 \leqslant i \leqslant n-1} \delta\left(p_{i}, t\left(a_{0} \cdots a_{i-1}\right), q_{i}^{0}, q_{i}^{1}\right) \\
& =\prod_{0 \leqslant i \leqslant n-1}^{0 \leqslant i \leqslant n-1} \frac{1}{2} \delta\left(p_{i}, t\left(a_{0} \cdots a_{i-1}\right), q_{i}^{0}, q_{i}^{1}\right) \\
& =\mu_{\mathcal{M}_{\mathcal{A}, t}}(\operatorname{Cyl}(\theta))
\end{aligned}
$$

\subsection{Decidability and Undecidability Results}

In this section, we show that the emptiness problem for probabilistic Büchi tree automata is decidable for the qualitative semantics for runs. We reduce this problem to deciding almost-surely winning in a POMDP, and the reduction works for any $\omega$-regular acceptance condition. However, the corresponding decision problem on POMDPs is only decidable for the Büchi condition. Hence we only obtain 
decidability in the Büchi case.

Let $\mathcal{A}=\left\langle A, Q, q_{\text {ini }}, \delta\right.$, Acc $\rangle$ be a probabilistic automaton with an $\omega$-regular acceptance condition and let $\Delta=Q \times Q \times Q$.

Define a POMDP $\mathbb{G}_{\mathcal{A}}=(\mathcal{G}, \sim, \mathcal{O})$ as follows. The arena $\mathcal{G}$ is equal to $\left\langle S, s_{\text {ini }}, A, \zeta\right\rangle$ where $S=Q \times\{0,1, \perp\} \times(\Delta \cup\{\perp\}), s_{\text {ini }}=\left(q_{0}, \perp, \perp\right)$ and $\zeta$ is defined as follows. For all $a \in A$ and $(p, x, t) \in S, \zeta((p, x, t), a)$ is the distribution that assigns $\frac{1}{2} \delta\left(p, a, q_{0}, q_{1}\right)$ to $\left(q_{y}, y,\left(p, q_{0}, q_{1}\right)\right)$ where $y=0,1$ and 0 to all other state. The objective $\mathcal{O}$ is the set of plays for which the sequence of states obtained when projecting on the first component belongs to Acc. The equivalence $\sim$ is defined by $(q, x, t) \sim\left(q^{\prime}, x^{\prime}, t^{\prime}\right)$ iff $x=x^{\prime}$.Intuitively in $\mathbb{G}_{\mathcal{A}}$, Éloïse describes a branch along a tree and Random builds a piece of run along this branch. As Éloïse does not observe the state in the run constructed by Random, it does not influence her choice for the branch.

TheOREM 46. Let $\mathcal{A}$ be a probabilistic tree automaton with an $\omega$-regular acceptance condition. The language $L_{\mathrm{Qual}}^{=1}(\mathcal{A})$ is non-empty if and only if Éloïse almostsurely wins in $\mathbb{G}_{\mathcal{A}}$.

Proof. From the definitions, we easily remark that $\{[\lambda] \sim \mid \lambda \in$ Plays $\}$ is in bijection with $=\{0,1\}^{*}$. Hence, strategies are in bijection with $A$-labeled trees. Once such a strategy $\varphi_{t}$ (seen as a tree $t$ ) is fixed, the resulting Markov chain is, up to renaming, $\mathbb{G}_{\mathcal{A}, t}$, meaning that the value of $\varphi_{t}$ is the value of $\mathbb{G}_{\mathcal{A}, t}$. In particular, Éloïse almost-surely wins in $\mathbb{G}_{\mathcal{A}}$ iff there is some $t$ such that $\operatorname{Val}\left(\mathbb{G}_{\mathcal{A}, t}\right)=1$ iff $t \in L_{\mathrm{Qual}}^{=1}(\mathcal{A})$ (thanks to Proposition 45).

For the Büchi acceptance condition, this leads a decidability result for the emptiness problem.

Corollary 47. Let $\mathcal{A}$ be a probabilistic Büchi tree automaton. Deciding emptiness of $L_{\mathrm{Qual}}^{=1}(\mathcal{A})$ is an EXPTIME-complete problem. Moreover, if $L_{\mathrm{Qual}}^{=1}(\mathcal{A}) \neq \varnothing$, it contains a regular tree.

Proof. The ExpTime upper-bound follows from the polynomial time reduction to deciding almost-surely winning in a Büchi POMDP. The lower bound follows from Proposition 43: emptiness of probabilistic Büchi $\omega$-word automata with the almost-sure acceptation (which is ExPTIME-complete [Baier et al. 2008; Baier et al. 2012]) can be reduced to our problem. Indeed, if $\mathcal{B}$ is a probabilistic Büchi $\omega$-word automaton we can construct a probabilistic Büchi tree automaton $\mathcal{A}$ of linear size (cf. Proposition 43) such that $L_{\text {Qual }}^{=1}(\mathcal{A})$ is equal to $\operatorname{Path}^{=1}\left(L^{=1}(\mathcal{B})\right)$ and in particular $L_{\text {Qual }}^{=1}(\mathcal{A})$ is empty if and only if $L^{=1}(\mathcal{B})$ is empty.

The proof of Theorem 46 together with the fact that a finite memory optimal strategy always exists in POMDP imply the existence of a regular tree when $L_{\text {Qual }}^{=1}(\mathcal{A}) \neq \varnothing$.

On the negative side, we show that the emptiness problem for probabilistic coBüchi tree automata is undecidable for both the classical and qualitative semantics for runs. These results are obtained by reduction to the undecidability of the emptiness problem for co-Büchi $\omega$-word automata with the almost-sure acceptation [Baier et al. 2008].

THEOREM 48. The following problems are undecidable: 
(1) given a probabilistic co-Büchi tree automaton $\mathcal{A}$, decide if $L^{=1}(\mathcal{A})=\varnothing$,

(2) given a probabilistic co-Büchi tree automaton $\mathcal{A}$, decide if $L_{\mathrm{Qual}}^{=1}(\mathcal{A})=\varnothing$.

Proof. Both undecidability results are shown by reduction to the undecidability of the emptiness problem for co-Büchi $\omega$-word automata (with the almost-sure acceptation).

Let $\mathcal{B}=\left\langle A=\{\mathrm{a}, \mathrm{b}\}, Q, q_{\text {ini }}, \delta, F\right\rangle$ be a probabilistic co-Büchi $\omega$-word automaton.

(1) We construct a probabilistic co-Büchi tree automaton $\mathcal{A}$ which simulates $\mathcal{B}$ on the left-most branch of the tree and checks that all other branches contain only a. Formally $\mathcal{A}$ is equal to $\left\langle A, Q \cup\left\{q_{a}, q_{\perp}\right\}, q_{\text {ini }}, \delta^{\prime}, F \cup\left\{q_{\perp}\right\}\right\rangle$. The probability distribution $\delta^{\prime}$ is given by:

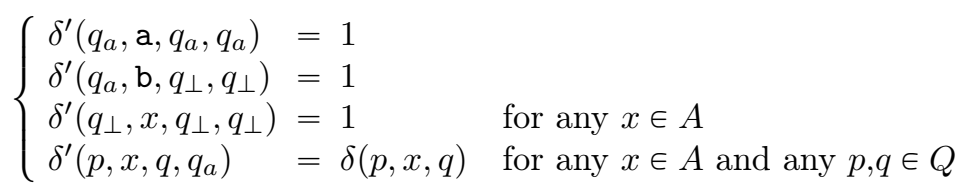

In all other cases, $\delta^{\prime}$ is equal to 0.

We claim that a tree $t$ belongs to $L^{=1}(\mathcal{A})$ if and only if for all $u \notin 0^{*}, t(u)=a$ and $t\left(0^{\omega}\right) \in L^{=1}(\mathcal{B})$. In particular $L^{=1}(\mathcal{A})$ is empty if and only if $L^{=1}(\mathcal{B})$ is empty.

Let $t$ be a tree accepted by $L^{=1}(\mathcal{A})$. Toward a contradiction assume that for some $u \notin 0^{*}, t(u)=b$. All accepting runs $\rho \in \operatorname{AccRuns}(\mathcal{A})$ are such that $\rho(u)=q_{a}$. In other terms, $\operatorname{AccRuns}(\mathcal{A})$ is included in the set $\{\rho \in \operatorname{Runs}(\mathcal{A}) \mid$ $\left.\rho(u)=q_{a}\right\}$ which has a null measure for $\mu_{t}$. Hence $\mu_{t}(\operatorname{AccRuns}(\mathcal{A}))=0$ which brings the contradiction.

We denote by $w$ the $\omega$-word over $\mathcal{A}$ corresponding to the left-most branch of $t$ (i.e. $\left.w=t\left(0^{\omega}\right)\right)$. Consider the mapping $p$ from $\operatorname{AccRuns}(\mathcal{A})$ to $\operatorname{AccRuns}(\mathcal{B})$ associating with a $\operatorname{run} \rho \in \operatorname{Runs}(\mathcal{A})$ the run $\rho\left(0^{\omega}\right)$.

For all partial run $\lambda=q_{0} q_{1} \ldots$ of $\mathcal{B}, p^{-1}(\mathrm{Cyl}(\lambda))$ is a finite union of cylinders corresponding to the (balanced) partial runs of $\mathcal{A}$ of depth $|\lambda|$ which coincide with $\lambda$ on their left-most branch. All these cylinders have measure 0 excepted the one corresponding to the partial run $\eta$ defined by $\eta(u)=q_{|u|}$ for $u \in 0 \leqslant|\lambda|$ and $\eta(u)=q_{a}$ otherwise. By construction of $\mathcal{A}, \mu_{w}(\operatorname{Cyl}(\lambda))=\mu_{t}(\operatorname{Cyl}(\eta))=$ $\mu_{t}\left(p^{-1}(\operatorname{Cyl}(\lambda))\right.$. This implies that $p$ is measurable and that the image of $\mu_{t}$ under $p$ is equal to $\mu_{w}$ using Carathéodory's unique extension theorem.

The other direction admits a similar proof.

(2) The case of qualitative semantics directly follows from Proposition 43. Indeed, let $\mathcal{A}$ be a probabilistic co-Büchi tree automaton such that $L_{\mathrm{Qual}}^{=1}(\mathcal{A})$ is equal to $\operatorname{Path}^{=1}\left(L^{=1}(\mathcal{B})\right)$. Then $L_{\text {Qual }}^{=1}(\mathcal{A})$ is empty if and only if $L^{=1}(\mathcal{B})$ is empty.

\subsection{Comparison with Regular Tree Languages and with Qualitative Tree Languages}

We now discuss expressiveness. First, we exhibit a family of tree languages that are accepted by a co-Büchi probabilistic automaton but that are neither regular tree languages nor qualitative tree languages. On the other hand, we give an 
example of a qualitative tree language that no probabilistic automaton (regardless of its semantics) can accept and another example of a regular tree language that no probabilistic automaton (regardless of its semantics) can accept. Hence, this proves incomparability of probabilistic tree languages with both qualitative and regular tree languages.

For the first result, we consider, for all $0<\lambda<1$, the $\omega$-word language $L_{\lambda}$ over $\{a, b\}$ defined by

$$
L_{\lambda}=\left\{a^{k_{1}} b a^{k_{2}} b \ldots \mid k_{1}, k_{2}, \ldots>0 \text { s.t. } \prod_{i=1}^{\infty}\left(1-\lambda^{k_{i}}\right)>0\right\}
$$

In [Baier et al. 2008], $L_{\lambda}$ is shown to be almost-surely accepted by a co-Büchi probabilistic automaton (actually they show that $L_{\lambda}$ is positively accepted by a Büchi automaton hence, as previously remarked in the introduction, is accepted by a co-Büchi probabilistic automaton). Therefore, by Proposition $43, \operatorname{Path}^{=1}\left(L_{\lambda}\right)$ is a co-Büchi probabilistic qualitative tree language.

Proposition 49. For all $0<\lambda<1$, $\mathrm{Path}^{=1}\left(L_{\lambda}\right)$ is neither a regular tree language nor a qualitative tree language.

Proof. Let $0<\lambda<1$. We will show that $\operatorname{Path}^{=1}\left(L_{\lambda}\right)$ does not contain any regular tree. Hence, thanks to Theorem 2 it is not a regular tree language and thanks to Corollary 18 it is not a qualitative tree language.

Toward a contradiction, assume that $L_{\lambda}$ contains a regular tree $t_{0}$. Consider the Markov chain $\mathcal{M}$ whose arena $\mathcal{G}$ is given by $\left(S, s_{\text {ini }}, \zeta\right)$. The set of states $S$ is the finite set of subtrees of $t$. The initial state $s_{\text {ini }}$ is $t_{0}$. The transition function $\zeta$ is defined for all $t \in S$ by $\zeta(t)(t[0])=\zeta(t)(t[1])=\frac{1}{2}$ and 0 otherwise. Clearly there exists a measure preserving bijection between the traces of $\mathcal{M}$ and the branchs of $t_{0}$.

To define the objective of the $\mathcal{M}$, consider the mapping $\psi$ for $S$ to $\{\mathrm{a}, \mathrm{b}\}$ associating to a state $t$ the label of its root $t(\varepsilon)$. This mapping can canonically be extended to associate a trace of $\mathcal{M}$ an $\omega$-word over $\{\mathrm{a}, \mathrm{b}\}$. The objective $\mathcal{O}$ of $\mathcal{M}$ is the set of traces whose image under $\psi$ belongs to $L_{\lambda}$.

As $t_{0}$ belongs to $\mathrm{Path}^{=1}\left(L_{\lambda}\right), \mathcal{M}$ almost-surely fulfils its objective.

We claim that there exists some $k>0$ such that the following event has a strictly positive probability: "The Markov chain from its current state reaches a state $t$ with $\psi(t)=\mathrm{b}$ and in less that $k$ steps another state $t^{\prime}$ with $\psi\left(t^{\prime}\right)=\mathrm{b}$."

Indeed, as $t_{0}$ belongs to $\mathrm{Path}^{=1}\left(L_{\lambda}\right)$, for all $t \in S$ there exists $u \sqsubseteq v$ such that $t(u)=t(v)=\mathrm{b}$. Hence, it suffices to take $k$ to be the maximum over all $t$ of the minimum of $|v|-|u|$ over all $u \sqsubseteq v$ such that $t(u)=t(v)=\mathrm{b}$.

By Borel-Cantelli lemma, this event occurs infinitely often along a trace with probability 1 . In particular, this implies that with probability 1 for a trace $\theta$ with $\psi(\theta)=a^{k_{1}} b a^{k_{2}} b \ldots \mid k_{1}, k_{2}, \ldots>0$ there are infinitely many $k_{i}$ 's which are equal to $k^{\prime} \leqslant k$. Hence $\psi(\theta)$ does not belongs to $L_{\lambda}$ (as for all $n>0, \prod_{i=1}^{\infty}\left(1-\lambda^{k_{i}}\right) \leqslant$ $\left.\left(1-\lambda^{k^{\prime}}\right)^{n}\right)$. This contradicts the fact that $\mathcal{M}$ fulfils its objective with probability 1.

REMARK 50. Using the correspondence with POMDP introduced in Theorem 46 , ACM Transactions on Computational Logic, Vol. V, No. N, Month 20 YY. 
any co-Büchi automaton accepting $\mathrm{Path}^{=1}\left(L_{\lambda}\right)$ gives rise to an example of a coBüchi POMDP $\mathbb{G}_{\mathcal{A}}$ in which Élö̈se needs infinite memory to almost-surely win.

We now give an example of a qualitative tree language that no probabilistic tree automaton can accept. For this we consider the language $\mathcal{L}_{\mathrm{a}}^{0 \vee 1}$ of $\{\mathrm{a}, \mathrm{b}\}$-labelled trees such that either the left subtree or the right subtree of the root belongs to the language $\mathcal{L}_{\mathrm{a}}$ of example 7 (recall that $\mathcal{L}_{\mathrm{a}}$ is the language of $\{\mathrm{a}, \mathrm{b}\}$-labelled trees whose set of branches containing at least one a has measure 1). Formally $\mathcal{L}_{\mathrm{a}}^{0 \vee 1}=\left\{t \mid t[0] \in \mathcal{L}_{\mathrm{a}}\right.$ or $\left.t[1] \in \mathcal{L}_{\mathrm{a}}\right\}$. One easily verifies that $\mathcal{L}_{\mathrm{a}}^{0 \vee 1}$ is a qualitative tree language. However, $\mathcal{L}_{\mathrm{a}}^{0 \vee 1}$ cannot be recognised by a probabilistic tree automaton.

Proposition 51. The language $\mathcal{L}_{a}^{0 \vee 1}$ cannot be recognised by a probabilistic tree automaton (regardless of its semantics).

Proof. By contradiction assume that there is some probabilistic parity tree automaton $\mathcal{A}=\left\langle\{\mathrm{a}, \mathrm{b}\}, Q, q_{\text {ini }}, \delta, \mathrm{Col}\right\rangle$ such that $\mathcal{L}_{\mathrm{a}}^{0 \vee 1}=L_{\text {Qual }}^{=1}(\mathcal{A})$ (the proof going exactly the same for the case where $\mathcal{L}_{\mathrm{a}}^{0 \vee 1}=L^{=1}(\mathcal{A})$ we omit it). Let $t_{\mathrm{a}}\left(\right.$ resp. $\left.t_{\mathrm{b}}\right)$ be the tree whose nodes are all labeled by a $($ resp. $\mathrm{b})$, i.e. $t_{\mathrm{a}}(u)=\mathrm{a}\left(\operatorname{resp}_{\mathrm{b}} t_{\mathrm{b}}(u)=\mathrm{b}\right)$ for all node $u \in\{0,1\}^{*}$. Now let $t_{0}$ be the tree defined by $t_{0}(\varepsilon)=\mathrm{b}, t_{0}[0]=t_{\mathrm{a}}$ and $t_{0}[1]=t_{\mathrm{b}}$; and $t_{1}$ be the tree defined by $t_{1}(\varepsilon)=\mathrm{b}, t_{1}[0]=t_{\mathrm{b}}$ and $t_{1}[1]=t_{\mathrm{a}}$. Obviously, both $t_{0}$ and $t_{1}$ belong to $\mathcal{L}_{\mathrm{a}}^{0 \vee 1}=L_{\text {Qual }}^{=1}(\mathcal{A})$.

For any state $q \in Q$, let $\mathcal{A}_{q}=\langle\{\mathrm{a}, \mathrm{b}\}, Q, q, \delta, \mathrm{Col}\rangle$ be the automaton obtained from $\mathcal{A}$ by changing its initial state to be $q$. Then one easily checks that a tree $t$ belongs to $L_{\text {Qual }}^{=1}(\mathcal{A})$ if and only if for all $\left(q_{0}, q_{1}\right) \in Q^{2}$ such that $\delta\left(q_{\text {ini }}, t(\varepsilon), q_{0}, q_{1}\right)>0$ one has $t[0] \in L_{\text {Qual }}^{=1}\left(\mathcal{A}_{q_{0}}\right)$ and $t[1] \in L_{\text {Qual }}^{=1}\left(\mathcal{A}_{q_{1}}\right)$.

Now, for any $\left(q_{0}, q_{1}\right) \in Q^{2}$ such that $\delta\left(q_{\text {ini }}, \mathrm{b}, q_{0}, q_{1}\right)>0$ one has that $t_{\mathrm{b}} \in$ $L_{\text {Qual }}^{=1}\left(\mathcal{A}_{q_{0}}\right)$ (because of $\left.t_{1} \in L_{\mathrm{Qual}}^{=1}(\mathcal{A})\right)$ and $t_{\mathrm{b}} \in L_{\mathrm{Qual}}^{=1}\left(\mathcal{A}_{q_{1}}\right)$ (because of $t_{0} \in$ $\left.L_{\mathrm{Q} \text { ual }}^{=1}(\mathcal{A})\right)$. Therefore the tree $t$ defined by $t(\varepsilon)=\mathrm{b}$ and $t[0]=t[1]=t_{\mathrm{b}}$ belongs to $L_{\mathrm{Qual}}^{=1}(\mathcal{A})$, which leads a contradiction as $t=t_{\mathrm{b}}$ and $t_{\mathrm{b}} \notin \mathcal{L}_{\mathrm{a}}^{0 \vee 1}=L_{\mathrm{Qual}}^{=1}(\mathcal{A})$.

We now give an example of a regular tree language that no probabilistic tree automaton can accept. For this, let $t_{\mathrm{a}}\left(\right.$ resp. $\left.t_{\mathrm{b}}\right)$ be the tree whose nodes are all labeled by a $($ resp. $\mathrm{b})$, i.e. $t_{\mathrm{a}}(u)=\mathrm{a}\left(\right.$ resp. $\left.t_{\mathrm{b}}(u)=\mathrm{b}\right)$ for all node $u \in\{0,1\}^{*}$. Let $t_{0}$ be the tree defined by $t_{0}(\varepsilon)=\mathrm{b}, t_{0}[0]=t_{\mathrm{a}}$ and $t_{0}[1]=t_{\mathrm{b}}$; and $t_{1}$ be the tree defined by $t_{1}(\varepsilon)=\mathrm{b}, t_{1}[0]=t_{\mathrm{b}}$ and $t_{1}[1]=t_{\mathrm{a}}$. Finally let $L_{0 \vee 1}=\left\{t_{0}, t_{1}\right\}$ : it is a regular tree language as it consists of two regular trees. However, $\mathcal{L}_{0 \vee 1}$ cannot be recognised by a probabilistic tree automaton.

Proposition 52. The language $\mathcal{L}_{0 \vee 1}$ cannot be recognised by a probabilistic tree automaton (regardless of its semantics).

Proof. One reasons by contradiction exactly as for Proposition 51 and concludes similarly that if there is a probabilistic automaton accepting $\mathcal{L}_{0 \vee 1}$ then it also accepts tree $t_{\mathrm{b}} \notin \mathcal{L}_{0 \vee 1}$, which leads a contradiction.

\subsection{Variants}

A natural variant is to replace the almost-sure acceptance condition on the set of runs by the probable one. That is a tree $t$ is probably accepted by $\mathcal{A}$ if $\mu_{t}(\operatorname{AccRuns}(\mathcal{A}))>0$.

ACM Transactions on Computational Logic, Vol. V, No. N, Month 20YY. 
Combining the conditions on the set of runs - almost-sure $(=1)$ and probable $(>0)$ - with the one on the set of accepting branches - qualitative (Qual) and positive (Pos) - we obtain four semantics for probabilistic tree automata denoted by $(>0$, Qual $),(>0$, Pos $),(=1$, Qual $)$ and $(=1$, Pos $)$ where the first component corresponds to the requirement on the set of accepting runs and the second to the requirement on the set of accepting branches of a run.

In Section 4, we mainly dealt with (=1, Qual)-probabilistic automata which have a tight link with POMDP for the almost-sure winning condition (cf. Theorem 46). It can be shown that ( $>0, \mathrm{Pos})$-probabilistic automata share the same connection with POMDP with the positive winning condition. It implies that the emptiness problem for the (>0, Pos)-probabilistic automata with a co-Büchi acceptance condition is ExpTime-complete.

When the two conditions are not of the same nature (as for the (>0, Qual) and $(=1$, Pos $)$ semantics $)$, we were unable to define a proper acceptance game.

We now briefly discuss properties of ( $>0$, Pos)-probabilistic automata. If $\mathcal{A}$ is a probabilistic tree automaton with an $\omega$-regular acceptance condition, we denote by QualAccRuns ${ }^{>0}(\mathcal{A})$ the set of positively accepting runs of $\mathcal{A}$ and by $L_{\text {Pos }}^{>0}(\mathcal{A})$ the set of trees accepted by $\mathcal{A}$ with $(>0$, Pos)-semantics. The proposition below (similar to Proposition 38) justifies of the definition of $L_{\mathrm{Pos}}^{>0}(\mathcal{A})$.

Proposition 53. For all probabilistic tree automata $\mathcal{A}$ with an $\omega$-regular acceptance condition the set QualAccRuns ${ }^{>0}(\mathcal{A})$ is measurable.

Proof. From Lemma 36 we have that $\operatorname{AccPairs}(\mathcal{A})=\{(\rho, \pi) \mid \rho(\pi) \in \operatorname{Acc}\}=$ $f_{\mathcal{A}}^{-1}(\{1\})$ is measurable in the product space. Hence the numerical function $g$ : Runs $\rightarrow[0,1]$ associating to a run $\rho$ the measure of its set of accepting branches (i.e. $\mu\left(\operatorname{AccPairs}(\mathcal{A})_{\rho}\right)$ where $\left.\operatorname{AccPairs}(\mathcal{A})_{\rho}=\{\lambda \in \operatorname{Br} \mid \rho(\lambda) \in \operatorname{Acc}\}\right)$ is measurable (cf. [Bauer 2001, Lemma 23.1 - p. 135]). As QualAccRuns ${ }^{>0}(\mathcal{A})$ is equal to $\left.\left.g^{-1}(] 0,1\right]\right)$, it is measurable.

The following proposition is an adaptation of Proposition 42 to the setting of (>0, Pos)-probabilistic automata.

Proposition 54. Let $\mathcal{A}$ be a probabilistic tree automaton with an $\omega$-regular acceptance condition and let $t$ be a tree. We have

$$
t \in L_{\mathrm{Pos}}^{>0}(\mathcal{A}) \Leftrightarrow \int f_{\mathcal{A}} d \mu_{t} \otimes \mu>0
$$

Proof. As $f_{\mathcal{A}}$ is measurable (Lemma 36), by Tonelli's theorem [Bauer 2001, Theorem 23.6 - p. 138], the mapping $g:$ Runs $\rightarrow[0,1]$ associating to a run $\rho \in$ Runs the value $\int_{\mathrm{Br}} f_{\mathcal{A}}(\rho, \cdot) d \mu$ is measurable.

$$
\begin{array}{ll} 
& t \text { belongs to } L_{\text {Pos }}^{>0}(\mathcal{A}) \\
\text { iff } & \left.\left.\mu_{t}\left(g^{-1}(\{] 0,1]\right\}\right)\right)>0 \\
\text { iff } & \int_{\text {Runs }} g d \mu_{t}>0 \\
\text { iff } & \int_{\text {Runs }} \int_{\mathrm{Br}} f_{\mathcal{A}} d \mu d \mu_{t}>0 \\
\text { iff } & \int_{\text {Runs } \times \operatorname{Br}} f_{\mathcal{A}} d \mu_{t} \otimes \mu>0
\end{array}
$$

By [Bauer 2001, Thm 13.2 - p. 71]

by definition of $g$

by Tonelli's theorem

[Bauer 2001, Theorem 23.6 - p. 138] 
Similarly to Proposition 43, we obtain a connection with $\omega$-word languages.

For an $\omega$-word language $L \subseteq\{\mathrm{a}, \mathrm{b}\}^{\omega}$, we denote by $\operatorname{Path}^{>0}(L)$ the set of trees labeled by $\{\mathrm{a}, \mathrm{b}\}$ with a positive set of branch labels in $L$ (i.e. $\mu(\{\pi \in \mathrm{Br} \mid t(\pi) \in$ $L\})>0$ ). In the following statement, for a probabilistic word automaton $\mathcal{B}$, we denote by $L^{>0}(\mathcal{B})$ the language positively-accepted by $\mathcal{B}$.

Proposition 55. Given a probabilistic $\omega$-word automaton $\mathcal{B}$ with an $\omega$-regular acceptance condition, there exists a probabilistic tree automaton $\mathcal{A}$ with the same acceptance condition such that $L_{\mathrm{Pos}}^{>0}(\mathcal{A})$ is equal to $\operatorname{Path}^{>0}\left(L^{>0}(\mathcal{B})\right)$.

Proof. Let $\mathcal{B}=\left\langle A, Q, q_{\text {ini }}, \delta\right.$, Acc $\rangle$ be a (complete) probabilistic $\omega$-word automaton with an $\omega$-regular condition. Consider the probabilistic tree automaton $\mathcal{A}$ simulating $\mathcal{B}$ on all branches. Formally $\mathcal{A}$ is equal to $\left\langle A, Q, q_{\text {ini }}, \delta^{\prime}, F\right\rangle$ where, for all $p, q \in Q$ and $x \in A, \delta^{\prime}(p, x, q, q)=\delta(p, x, q)$.

Let $t$ be a tree and let $g: \mathrm{Br} \mapsto[0,1]$ be the mapping associating to a branch $\pi$ the value $\int f_{\mathcal{A}}(\cdot, \pi) d \mu_{t}$. We have the following claim whose proof is identical to that of Claim 44 in Proposition 43.

Claim 56. For any $\pi \in \mathrm{Br}, g(\pi)>0$ if and only if $t(\pi)$ belongs to $L^{>0}(\mathcal{B})$.

We are now ready to conclude:

$$
\begin{aligned}
& t \in L_{\mathrm{Pos}}^{>0}(\mathcal{A}) \\
& \Leftrightarrow \int f_{\mathcal{A}} d \mu_{t} \otimes \mu>0 \quad \text { Proposition } 54 \\
& \Leftrightarrow \int g d \mu=1 \quad \text { Tonelli's Theorem } \\
& \Leftrightarrow g(\pi)>0 \text { on a non-null set [Bauer2001, Theorem } 13.2-p .71 \text { ] } \\
& \Leftrightarrow \mu\left(\left\{\pi \mid t(\pi) \in L^{>0}(\mathcal{B})\right\}\right)>0 \quad \text { Claim } 56 \\
& \Leftrightarrow t \in \operatorname{Path}^{>0}\left(L^{>0}(\mathcal{B})\right)
\end{aligned}
$$

We can also transfer the results on the acceptance game (Proposition 45 and Theorem 46).

Proposition 57. Let $\mathcal{A}$ be a probabilistic tree automaton with an $\omega$-regular acceptance condition and let $t$ be a tree. We have $t \in L_{\mathrm{Pos}}^{>0}(\mathcal{A})$ iff $\mathcal{M}_{\mathcal{A}, t}$ positively fulfils its objective.

Proof. Let AccPairs $(\mathcal{A})=\{(\rho, \pi) \mid \rho(\pi) \in \operatorname{Acc}\}$. By Proposition 54, to establish the desired equivalence, it is sufficient to show that $\mu_{t} \otimes \mu(\operatorname{AccPairs}(\mathcal{A}))=$ $\mu_{\mathcal{M}_{\mathcal{A}, t}}\left(\mathcal{O}_{\text {Acc }}\right)$. The latter was done already in the proof of Proposition 45.

TheOREM 58. Let $\mathcal{A}$ be a probabilistic tree automaton with an $\omega$-regular acceptance condition. The language $L_{\mathrm{Pos}}^{>0}(\mathcal{A})$ is non-empty if and only if Éloïse positively wins in $\mathbb{G}_{\mathcal{A}}$.

Proof. The proof only differs from the one of Theorem 46 in its conclusion. Namely, Éloïse positively wins in $\mathbb{G}_{\mathcal{A}}$ iff there is some $t$ such that $\operatorname{Val}\left(\mathbb{G}_{\mathcal{A}, t}\right)>0$ iff $t \in L_{\mathrm{Pos}}^{>0}(\mathcal{A})$ (thanks to Proposition 57).

If we consider a co-Büchi acceptance condition, this leads a decidability result for the emptiness problem (which is a dual version of Corollary 47).

ACM Transactions on Computational Logic, Vol. V, No. N, Month 20YY. 
Corollary 59. Let $\mathcal{A}$ be a probabilistic co-Büchi tree automaton. Deciding emptiness of $L_{\mathrm{Pos}}^{>0}(\mathcal{A})$ is an EXPTIME-complete problem. Moreover, if $L_{\mathrm{Pos}}^{>0}(\mathcal{A}) \neq$ $\varnothing$, it contains a regular tree.

Proof. The ExpTime upper-bound follows from the polynomial time reduction to deciding positive winning in a co-Büchi POMDP. The proof of Theorem 58 together with the fact that a finite memory positively winning strategy always exists in POMDP imply the existence of a regular tree when $L_{\mathrm{Pos}}^{>0}(\mathcal{A}) \neq \varnothing$.

The lower bound can be establish as for Corollary 47 by reduction of the emptiness problem of probabilistic co-Büchi $\omega$-word automata with the probable semantics (which is ExpTime-complete [Baier et al. 2008; Baier et al. 2012]). For this one first establishes a dual version of Proposition 43 and then concludes as in Corollary 47.

Finally, as a dual version of Theorem 48, we show that the emptiness problem for a Büchi acceptance condition is undecidable.

THEOREM 60. The following problem is undecidable : given a probabilistic Büchi tree automaton $\mathcal{A}$, decide if $L_{\mathrm{Pos}}^{>0}(\mathcal{A})=\varnothing$.

Proof. The proof proceeds by reduction to the undecidability of the emptiness problem for Büchi $\omega$-word automata (with the positive acceptation).

Let $\mathcal{B}=\left\langle A=\{\mathrm{a}, \mathrm{b}\}, Q, q_{i n i}, \delta, F\right\rangle$ be a probabilistic Büchi $\omega$-word automaton. By Proposition 55, we can construct a probabilistic Büchi tree automaton $\mathcal{A}$ such that:

$$
L_{\mathrm{Pos}}^{>0}(\mathcal{A})=\operatorname{Path}^{>0}\left(L^{>0}(\mathcal{B})\right) .
$$

In particular $L_{\mathrm{Pos}}^{>0}(\mathcal{A})$ is empty if and only if $L^{>0}(\mathcal{B})$ is empty as well, which allows us to conclude.

We terminate this Section with Table I that summarises the (un)decidability results and open questions on the emptiness problem for the different types of probabilistic semantics that we considered.

\begin{tabular}{|c|c|c|c|c|}
\hline & $L^{=1}(\mathcal{A})$ & $L_{\text {Qual }}^{=1}(\mathcal{A})$ & $L_{\text {Pos }}^{>0}(\mathcal{A})$ & $\begin{array}{c}L_{\text {Pos }}^{=1}(\mathcal{A}) / \\
L_{\text {Qual }}^{>0}(\mathcal{A})\end{array}$ \\
\hline Büchi & Open & $\begin{array}{c}\text { ExpTIME- } \\
\text { complete } \\
\text { Corollary 47) }\end{array}$ & $\begin{array}{c}\text { Undecidable } \\
(\text { Theorem 60) }\end{array}$ & Open \\
\hline co-Büchi & $\begin{array}{c}\text { Undecidable } \\
\text { Theorem 48) }\end{array}$ & $\begin{array}{c}\text { Undecidable } \\
\text { Theorem 48) }\end{array}$ & $\begin{array}{c}\text { ExPTIME- } \\
\text { complete } \\
\text { Corollary 59) }\end{array}$ & Open \\
\hline
\end{tabular}

Table I. Decidability status of the emptiness problem for the different types of probabilistic semantics. 


\section{CONCLUSION}

The first main contribution of this paper was to introduce new acceptance criteria for non-deterministic automata on infinite trees: qualitative and positive acceptance. Both criteria define (incomparable) classes of languages with desirable closure and decidability properties (in particular a polynomial time emptiness test). In addition, it leads to a tight connection with (finite) Markov decision processes in a similar flavour as regular tree automata do with two-player games.

The second main contribution was to define suitable notions of probabilistic automata. In particular we showed that for Büchi (=1, Qual)-probabilistic automata as well as co-Büchi ( $>0$, Pos)-probabilistic automata emptiness is an ExPTIMEcomplete problem.

Unsurprisingly, there remain several open questions. Some of them are purely theoretical (mainly regading expressiveness and decidability status of the emptiness problem for some variants) but the most pressing one concerns potential applications of this work. A quick answer to this latter challenge is to rely on the tight connection between qualitative tree languages and Markov decision processes as exposed in Section 3.5. As these two objects are essentially the same one seen from two different perspectives (the qualitative tree languages being a sort of unfolding of a finite MDP) one can for instance rely on the modelling work made using MDP (see e.g. [Baier and Katoen 2008, Chapter 10] for many valuable examples) to argue that qualitative tree languages are equally useful for such a purpose. However, the setting of MDP seems simpler for modelling purpose as it is closer to real systems. Concerning the probabilistic setting, it is not clear yet what are the potential applications. Due to their incomparability with both regular and qualitative tree languages, we cannot simply extend existing applications. But this is also encouraging as it suggest potentially new applications. The hard part being that it mixes (for the qualitative semantics) two orthogonal notions of measure: the one on the run and the one the branches; if the one on branches has a simple interpretation (one looks at all possible executions of a system) the one on runs is more tricky to interpret (in a sense it speaks of all output of a machine processing the unfolding of a system).

\section{ACKNOWLEDGMENTS}

The authors would like to thank the anonymous reviewers for their thorough reviews and very useful comments that helped improve the clarity and the relevance of this paper.

This work was supported by the following projects: AMIS (ANR 2010 JCJC 0203 01 AMIS) and FREC (ANR 2010 BLAN 020202 FREC).

\section{REFERENCES}

Baier, C., Bertrand, N., and Grösser, M. M. 2008. On decision problems for probabilistic Büchi automata. In Proceedings of the 11th International Conference on Foundations of Software Science and Computation Structures (FoSSaCS 2008). Lecture Notes in Computer Science, vol. 4962. Springer, 287-301.

Baier, C., Grösser, M., and Bertrand, N. 2012. Probabilistic w-automata. Journal of the Association of Computing Machinery 59, 1, 1.

Baier, C. And Grösser, M. M. 2005. Recognizing omega-regular languages with probabilistic

ACM Transactions on Computational Logic, Vol. V, No. N, Month 20YY. 
automata. In Proceedings of the 20th IEEE Symposium on Logic in Computer Science (LiCS 2005). IEEE Computer Society, 137-146.

Baier, C. And Katoen, J.-P. 2008. Principles of Model Checking (Representation and Mind Series). The MIT Press.

Bauer, H. 1996. Probability Theory. Walter de Gruyter.

Bauer, H. 2001. Measure and Integration Theory. Walter de Gruyter.

Beauquier, D., Nivat, M., And Niwinski, D. 1991. About the effect of the number of successful paths in an infinite tree on the recognizability by a finite automaton with büchi conditions. In Proceedings of Fundamentals of Computation Theory, 8th International Symposium (FCT 1991). Lecture Notes in Computer Science, vol. 529. Springer, 136-145.

Beauquier, D. And Niwiński, D. 1995. Automata on infinite trees with path counting constraints. Information and Computation 120, 1, 117 - 125.

Bienvenu, L. 2008. Caractérisations de l'aléatoire par les jeux: impredictibilité et stochasticité. Ph.D. thesis, Université de Provence.

Chadha, R., Sistla, A. P., and Viswanathan, M. 2009. Power of randomization in automata on infinite strings. In Proceedings of Concurrency Theory, 20th International Conference (CONCUR 2009). Lecture Notes in Computer Science, vol. 5710. Springer, 229-243.

Chatterjee, K., Doyen, L., And Henzinger, T. A. 2009. Probabilistic weighted automata. In Proceedings of Concurrency Theory, 20th International Conference (CONCUR 2009). Lecture Notes in Computer Science, vol. 5710. Springer, 244-258.

Chatterjee, K., Jurdziński, M., And Henzinger, T. A. 2004. Quantitative stochastic parity games. In Proceedings of the Fifteenth Annual ACM-SIAM Symposium on Discrete Algorithms (SODA 2004). SIAM, 121-130.

Church, A. 1962. Logic, arithmetic and automata. In Proceedings of the International Congress of Mathematicians. 23-35.

Courcoubetis, C. And Yannakakis, M. 1990. Markov decision processes and regular events (extended abstract). In Proceedings of the 17th International Colloquium on Automata, Languages, and Programming (ICALP 1990). Lecture Notes in Computer Science, vol. 443. Springer, 336-349.

Emerson, E. A., Jutla, C. S., And Sistla, A. P. 1993. On model-checking for fragments of $\mu$-calculus. In Proceedings of the 5th International Conference on Computer Aided Verification (CAV 1993). Lecture Notes in Computer Science, vol. 697. Springer, 385-396.

Grädel, E., Thomas, W., And Wilke, T., Eds. 2002. Automata, Logics, and Infinite Games: A Guide to Current Research [outcome of a Dagstuhl seminar, February 2001]. Lecture Notes in Computer Science, vol. 2500. Springer.

Gurevich, Y. and Harrington, L. 1982. Trees, automata, and games. In Proceedings of the Fourteenth Annual ACM Symposium on Theory of Computing (STOC 1982). ACM, 60-65.

KaKutani, S. 1948. On equivalence of infinite product measures. Annals of Mathematics 49, $214-224$.

LöDing, C. 2011. Infinite games and automata theory. In Lectures in Game Theory for Computer Scientists, K. Apt and E. Grädel, Eds. Cambridge University Press, 38-73.

Muchnik, A. A., Semenov, A. L., And Uspensky, V. A. 1998. Mathematical metaphysics of randomness. Theoretical Computer Science 207, 2, 263-317.

PAz, A. 1971. Introduction to probabilistic automata. Academic Press New York.

Perrin, D. And Pin, J.-E. 2004. Infinite Words. Academic Press.

RABIN, M. O. 1963. Probabilistic automata. Information and Control 6, 3, 230-245.

RABin, M. O. 1969. Decidability of second-order theories and automata on infinite trees. Transaction of the American Mathematical Society 141, 1-35.

RABIN, M. O. 1972. Automata on infinite objects and Church's problem. American Mathematical Society, Providence, R.I. Conference Board of the Mathematical Sciences Regional Conference Series in Mathematics, No. 13.

Reisz, R. D. 1999. Decomposition theorems for probabilistic automata over infinite objects. Informatica, Lith. Acad. Sci. 10, 4, 427-440. 
Thomas, W. 1997. Languages, automata, and logic. In Handbook of Formal Language Theory, G. Rozenberg and A. Salomaa, Eds. Vol. III. Springer, 389-455.

VARdi, M. Y. AND Wilke, T. 2007. Automata: from logics to algorithms. In Logic and Automata: History and Perspectives. Amsterdam University Press, 629-736.

ACM Transactions on Computational Logic, Vol. V, No. N, Month 20YY. 